3

2

3

7

\title{
A Scalable, Open-Source Implementation of a Large-Scale Mechanistic Model for Single Cell Proliferation and Death Signaling
}

Cemal Erdem ${ }^{1,}$, Arnab Mutsuddy ${ }^{1}$, Ethan M. Bensman ${ }^{2}$, William B. Dodd ${ }^{1}$, Michael M. SaintAntoine $^{3}$, Mehdi Bouhaddou ${ }^{4}$, Robert C. Blake ${ }^{5}$, Sean M. Gross ${ }^{6}$, Laura M. Heiser ${ }^{6}$, F. Alex Feltus $^{7,8,9}$, and Marc R. Birtwistle ${ }^{1,10,{ }^{*}}$

${ }^{1}$ Department of Chemical \& Biomolecular Engineering, Clemson University, Clemson, SC

${ }^{2}$ Computer Science, School of Computing, Clemson University, Clemson, SC

${ }^{3}$ Center for Bioinformatics and Computational Biology, University of Delaware, Newark, DE

${ }^{4}$ Department of Cellular and Molecular Pharmacology, University of California San Francisco, San Francisco, CA

${ }^{5}$ Center for Applied Scientific Computing, Lawrence Livermore National Laboratory, Livermore, CA

${ }^{6}$ Department of Biomedical Engineering, Oregon Health \& Science University, Portland, OR

${ }^{7}$ Department of Genetics and Biochemistry, Clemson University, Clemson, SC

${ }^{8}$ Biomedical Data Science and Informatics Program, Clemson University, Clemson, SC

${ }^{9}$ Center for Human Genetics, Clemson University, Clemson, SC

${ }^{10}$ Department of Bioengineering, Clemson University, Clemson, SC

*Correspondence: cemalerdem@gmail.com (C.E.) and mbirtwi@clemson.edu (M.R.B.)

1

4

5

6




\section{ABSTRACT}

Mechanistic models of how single cells respond to different perturbagens can help integrate disparate big data sets or predict response to varied drug combinations. However, the construction and simulation of such models have proved challenging. Our lab previously constructed one of the largest mechanistic models for single mammalian cell regulation of proliferation and death (774 species, 141 genes, 8 ligands, 2400 reactions). However, this, as many other large-scale models, was written using licensed software (MATLAB) with intricate programming structure, impeding alteration, expansion, and sharing. Here, we generated a new foundation for this model, which includes a python-based creation and simulation pipeline converting a few structured text files into an SBML-compatible format. This new open-source model (named SPARCED) is high-performance- and cloud-computing compatible and enables the study of virtual cell population responses at the single-cell level. We applied this new model to a subset of the LINCS MCF10A Data Cube, which observed that IFN $\gamma$ acts as an antiproliferative factor, but the reasons why were unknown. After expanding the SPARCED model with an IFN $\gamma$ signaling module (to 950 species, 150 genes, 9 ligands, 2500 reactions), we ran stochastic single-cell simulations for two different putative crosstalk mechanisms and looked at

44 the number of cycling cells in each case. Our model-based analysis suggested, and experiments support that these observations are better explained by IFN $\gamma$-induced SOCS1 expression sequestering activated EGF receptors, thereby downregulating AKT activity, as opposed to direct

47 IFN $\gamma$-induced upregulation of p21 expression. This work forms a foundation for increased 48 mechanistic model-based data integration on a single-cell level, an important building block for 49 clinically predictive mechanistic models. 


\section{INTRODUCTION}

52 The ever-increasing availability and accumulation of FAIR (1) (findable, accessible, interoperable,

53 and reproducible) and big (omics) datasets requires new computational methods and models to

54 integrate, analyze, and interpret the underlying information (2-4). How can we leverage the totality

55 of available information not only to learn more about biology but also to make predictions,

56 especially those that are clinically relevant? Advances in statistical and machine learning

57 approaches enable (mostly) data-driven exploration and hypothesis generation from big datasets

58 (5-8). Trained on features of the input dataset(s), such models can be used for, as just a few

59 examples, to predict drug responses (9-11) or decide tumor type/stage (12-15). Although

60 transformative, such machine learning and statistical models have shortcomings. Most notably,

61 they often fail to explain predicted outcomes with detailed mechanistic reasoning (16-20) - a

major scientific gap and a roadblock to reconciling and integrating such models.

Besides such "black-box" modeling approaches, an alternative and complementary vehicle

for data integration are so-called "mechanistic models" (20). Mechanistic models provide an interpretable integration of different data types, because they have explicitly modeled biophysical correlates, while enabling further exploration for underlying logic behind heterogeneous, nonlinear, and often unintuitive relationships across big datasets (21). If mechanistic models are available towards the whole-genome or whole-single-cell scale, one can start to predict complex, multi-network, and emergent cellular behaviors $(22,23)$, elucidate phenotypic responses to 70 multiple perturbations $(24,25)$, tailor and train on patient-specific data for personalized, 71 pharmacologic decision making $(26,27)$, or use them as "data integrators" for data consistency 72 checking (28). However, most published mechanistic models are "small" scale; built for single pathways with a handful of genes, meant to interpret a single dataset (29-38). Such small-scale

74 mechanistic models provided important insights into processes such as yeast response to 
stimulations (29). However, the limited scope of small-scale models means they inherently will

77 struggle to integrate multiple datasets. Large-scale mechanistic models $(23,39-41)$, on the other

78 hand, can provide a more extensive representation of cellular interactions and are thus well-

79 poised for data integration that complement shortcomings of machine learning approaches.

One of the many ways of mechanistic model construction is the use and modification of existing models by inserting new species or interactions to explain new experimental observations $(38,42,43)$. Model merging, the act of stitching pre-existing models together, is an extension of this method for creating larger models. However, such an approach requires extensive detail checking and harmonizing species/parameter definitions. Often, unfortunately, sufficient annotation is not provided which makes this task harder. Moreover, while most mechanistic models are comprised of ordinary differential equations (ODEs), many large-scale models require multiple sub-modules of different mathematical formalisms. For example, metabolic processes are usually described by steady-state flux-balance models $(44,45)$, gene expression events are stochastic (46-48), and protein signaling events are represented by a system of ODEs $(29,30,38)$.

Thus, sorting out a single platform for different modeling formalisms to create a large-scale model

91 is a daunting task. It is so far only achieved by creating highly custom-structured and custom-

92 coded model-agglomerates that are not well-suited to further alterations or re-use $(23,40)$. The

93 latter, Bouhaddou2018 pan-cancer model (40), is previously published by our group to study

94 single-cell responses to mitogens and drugs.

A second way of constructing models is to build them bottom-up by writing out every reaction one by one. In this regard, rule-based modeling (RBM) provides an innovative approach (49). RBM software, such as BioNetGen (50,51), Kappa (52), and PySB (53), enables researchers to write "rules" for repeated reaction events following specific patterns. RBM software then creates 
101 writing out every differential equation), some limitations exist. First, it can generate a vast (even 102 infinite) number of reactions from a small set of rules (usually called the curse of combinatorial 103 complexity). This makes interpreting, analyzing, and debugging such models cumbersome, if 104 possible. Tools like NFsim (54) can overcome such problems by simulating events based on the 105 rules rather than a priori generating the entire reaction network. Thus, such software becomes 106 advantageous when a small number of rules create a very large number of reactions, e.g., 107 polymerization, aggregation, or multi-site phosphorylation (55). However, such network-free 108 simulators typically require an explicit representation of every molecule in the system, which 109 dramatically increases the computational cost and renders such methods inefficient for large110 scale mechanistic models. Secondly, current RBM implementations dictate that reactions taking 111 place via the same rule have the same rate constant parameter values. Often, allostery or site 112 cooperativity precludes this simplifying assumption, leading to manually writing out every such reaction in the model (or writing one rule for each reaction), which then obviates the advantages 114 of RBM. Finally, with its capability of capturing biological complexity via simple rules, the RBM concept is quite powerful but additional efforts are needed to enable merging of existing non-rulebased models, creating a mixture of different modeling formats (i.e. mixed-grain modeling), and defining different simulation settings (i.e. hybrid modeling = deterministic + stochastic parts). FAIRness (findable, accessible, interoperable, and reproducible) and re-useability (56-58). Porubsky et al. (57) recently summarized the best modeling practices and reinforced: providing 121 metadata/annotations and model creation steps/files (Practices 1-5), using standard and cross122 platform model files (Practice 3), and open-source, license-free, version-controlled, and 123 reproducible model dissemination (Practices 8-9). As the size of the model increases, conforming 124 to modeling standards (e.g. simulation type, simulation speed, software to use, scripting package 125 to use, algorithm to use) gets harder. That is why most of the large-scale (many genes or whole- 
126

127

128

129

130

131

132

133

134

135

136

137

138

139

140

141

142

143

144

145

cell) models are necessarily custom-structured, are composed of multiple submodules, or are lacking sufficient annotations and metadata (e.g. ENSEMBL or HGNC identifiers) (23,39-41). These custom-made models also do not yet follow a single standard format, a key property for easy distribution, re-use, and model merging and expansion with other models. The SBML (Systems Biology Markup Language) format $(59,60)$ offers a long-established and well-defined way of specifying annotated model structures, with an explicit and structured definition of each element of a mechanistic model (species, reactions, volumes, initial concentrations, parameters, rules, events, equations). SBML is an extensible, machine-readable markup language and not a simple text file. SBML has interfaces and packages in most programming languages (like Python, C++, Perl) and can be imported by most software (Python, MATLAB, COPASI (61), Virtual Cell (62), and another $\sim 300$ packages). However, it is non-trivial to write thousands of reactions in SBML standards, directly or with available GUI-based software. To circumvent this problem, there are efforts to convert other model formats to SBML, like Antimony (63). The Antimony format is defined in simple text format and is human readable and interpretable. Regardless, any constructed mechanistic model, in SBML format or not, must be simulated with reasonable CPU time. Although simulating models on local machines is often done, High Performance (HPC) or Cloud Computing (CC) platforms are suitable for larger tasks such as parameter sensitivity/estimation or multiple single-cell simulations (64-67). Therefore, another milestone for large-scale mechanistic models is inherent HPC/CC compatibility, especially for single-cell simulations and heterogeneous data integration.

Here, we provide a framework for large-scale mechanistic modeling that converts our lab's previous large-scale pan-cancer model into a format that conveys several crucial properties noted above. First, we define a simple set of structured and annotated input text files that set model specifics: genes, species, reactions, reaction stoichiometry, cellular compartments, transcriptional regulations, input omics data, and parameter values (Fig. 1). These text files enable easy creation 
or alteration of the model network, without any coding or software usage requirements (but they are easily amenable to such things if desired). We then use Jupyter notebooks (68) to process the input files and to create a human-interpretable Antimony file, which is then converted into an SBML (community gold-standard) model file. We simulate the model using SBML compatible Python packages including $\mathrm{AMICI}$, specifically designed for efficient simulation of large-scale models $(67,69)$, and our own Python submodule for stochastic gene expression that enables single-cell simulations. We also develop an HPC/CC (Kubernetes) compatible version of the pipeline that enables simulating large number of single cells and/or stimulation conditions. To apply our work, we re-create and extend our previous single mammalian cell mechanistic model of proliferation and death signaling and regulation (40), which we call SPARCED (SBML, expression upregulation. This large-scale mechanistic model construction framework and the SPARCED model outlined here is an important step towards creating and testing large-scale mechanistic models as data integration and clinical decision-making tools.

Fig 1. SPARCED is a structured, human interpretable, and easy to modify big mechanistic model. (A) The schematic of the underlying model for SPARCED. Image adapted from (40). (B) The pan-cancer mechanistic model Bouhaddou2018 is re-written in open-source and structured file format. The steps of model construction include input file creation and conversion into an SBML file. The optional initialization step calibrates model parameters for new cellular contexts and phenotypic behaviors. The annotated SBML model file and stochastic module are simulated together at single-cell level locally or by using cloud-computing. The benefits of the new SPARCED model include easy alteration and expansion capabilities through text file editing, 
bioRxiv preprint doi: https://doi.org/10.1101/2020.11.09.373407; this version posted July 15, 2021. The copyright holder for this preprint (which was not certified by peer review) is the author/funder. All rights reserved. No reuse allowed without permission.

180 simulation. The modeling pipeline introduced here are inline with good practices of re-usable big 181 mechanistic models (57). (C) The Bouhaddou2018 model file types are simplified and converted 182 into open-source platforms. 


\section{RESULTS}

\section{SPARCED Model Construction and Unit Testing}

185 Current large-scale mechanistic models are agglomerates of smaller models and tools, used 186 mainly within the same research lab. Most such models also lack clear and satisfactory annotation and metadata, making them harder to understand and alter $(23,40)$. The goals of this work were (i) to build tools that help large-scale mechanistic model construction and alteration, that is simple, efficient, open-source, and cloud computing compatible; (ii) to provide a scalable and re-useable big mechanistic model for a single mammalian cell; and (iii) demonstrate the work through application to a biological question.

We first created a set of simple input files and scalable processing scripts for one of the broadest cancer signaling models in the literature (40), called the Bouhaddou2018 model here (Fig. 1A). The input files (Supp. Files 1-7) are simple tab-separated text files (Fig. 1B-C), unlike licensed file formats with a mixture of hard coded information in multiple interconnected scripts commonly used in modeling literature. A Jupyter notebook (Supp. File 8) processes the input files

197 into an Antimony text file (Supp. File 9). The model creation code generates the SPARCED model 198 file in SBML format (Supp. File 10) using the Antimony text file and annotations from the model input files (Supp. Files 2 and 6). When the model construction step is complete and the SBML file is created, it is imported and simulated using a Python package called $\mathrm{AMICI}(67,69)$. For every new cell line model, a pre-calibration step called Initialization is employed to tune parameter values. Here, we ensure total protein levels match experimental observations and particular phenotypic criteria are met; for example, we specify that serum and growth factor starved cells on model is compiled for model testing and other simulations. 
The result is what should be a replica of the Bouhaddou2018 model, which we call SPARCED. Like the Bouhaddou2018 model, the initial SPARCED model is based on non-transformed breast epithelial MCF10A cell line data. We annotated all the species in the model with HGNC gene identifiers, providing easier programmatic filtering and curation of species list, while keeping the user defined simpler names for complicated species structures. However, the extent to which the models are congruent was not yet clear, and thus we next set out to examine agreement between the two. We verified that the previous Bouhaddou2018 model simulations are reproducible and match expected experimental observations through the same unit test concept (Table 1) introduced for the original model (40). Each unit test has a dedicated Jupyter notebook on GitHub repository (github.com/birtwistlelab/SPARCED/SPARCED_Brep). We illustrate select unit testing examples below, but all results are presented in supplementary figures (Figs. S2-11).

Table 1. List of SPARCED model unit testing and comparisons to Bouhaddou2018 model. The SPARCED model passed each test depicted below and recapitulated experimental and simulation observations reported by the Bouhaddou2018 model.

\section{Descriptions of unit tests}

Functional test to ensure the deterministic module is updated every 30 seconds with mRNA numbers generated by the stochastic module.

Simulated ligand-receptor cooperativity coefficients for the receptor tyrosine kinases match experimental observations (negative cooperativity: EGF, FGF, IGF, INS; no cooperativity: HGF, NRG1, and positive cooperativity: PDGF).

Activated EGF receptors internalize and peak $\sim 30$ minutes after ligand treatment.

EGF and insulin stimulation activates both ERK and AKT pathways. Dual stimulation with the two ligands induces prolonged AKT activation.

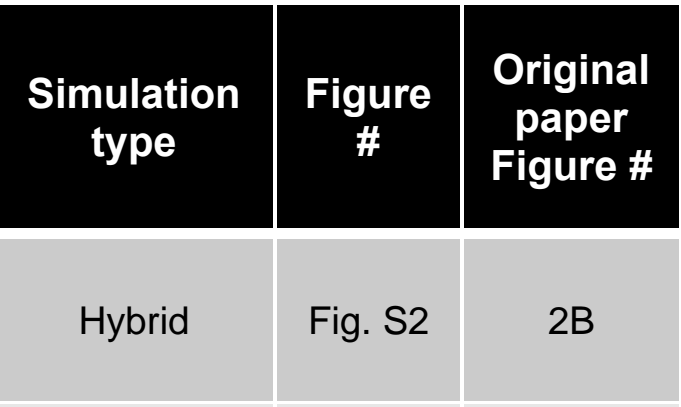

\begin{tabular}{l|l|l} 
Deterministic & Fig. & $3 \mathrm{~A}+\mathrm{S} 3 \mathrm{~A}$
\end{tabular}

Deterministic

Fig. S3B S3B

Deterministic

Figs. S4-5 $\mathrm{S} 3 \mathrm{C}$ 


\begin{tabular}{|c|c|c|c|}
\hline $\begin{array}{l}\text { Double and/or single stranded DNA damage } \\
\text { activates p53 and DNA damage repair mechanisms } \\
\text { represses its response. }\end{array}$ & Deterministic & $\begin{array}{l}\text { Fig. } \\
\text { S6A }\end{array}$ & $3 E$ \\
\hline $\begin{array}{l}\text { Increasing DNA damage amount in single cells leads } \\
\text { to higher number of activated p53 peaks. }\end{array}$ & Hybrid & $\begin{array}{l}\text { Fig. } \\
\text { S6B-C }\end{array}$ & $3 F+S 3 E$ \\
\hline $\begin{array}{l}\text { Increasing simulated TRAIL dose decreases the time } \\
\text { it takes to die for an average cell. }\end{array}$ & Deterministic & $\begin{array}{l}\text { Fig. } \\
\text { S7A-B }\end{array}$ & $3 G$ \\
\hline $\begin{array}{l}\text { The fraction of surviving cells decreases as } \\
\text { stimulated TRAIL dose increases. }\end{array}$ & Hybrid & $\begin{array}{l}\text { Fig. } \\
\text { S7C }\end{array}$ & $3 \mathrm{H}$ \\
\hline $\begin{array}{l}\text { Increasing ERK and AKT activity levels prolongs } \\
\text { TRAIL induced time to death, whereas increasing } \\
\text { PUMA and NOXA expression levels decreases the } \\
\text { time it takes for cells to die. }\end{array}$ & Deterministic & $\begin{array}{l}\text { Fig. } \\
\text { S7D }\end{array}$ & 31 \\
\hline $\begin{array}{l}\text { Increasing Cyclin D mRNA levels induces proper } \\
\text { cyclin-CDK complex progression and oscillations for } \\
\text { cell cycle entry and progression. }\end{array}$ & Deterministic & $\begin{array}{l}\text { Fig. } \\
\text { S8A }\end{array}$ & $3 \mathrm{~J}$ \\
\hline $\begin{array}{l}\text { Etoposide treatment induces cell cycle arrest and cell } \\
\text { death. Cycling cells (with prior growth factor } \\
\text { stimulation) show increased percentage of death to } \\
\text { etoposide treatment, compared to non-cycling cells. }\end{array}$ & Hybrid & $\begin{array}{l}\text { Fig. } \\
\text { 3D-E }\end{array}$ & $4 A, B, C$ \\
\hline $\begin{array}{l}\text { Inhibition of AKT and ERK pathways together } \\
\text { synergistically increase cell death, in EGF and insulin } \\
\text { stimulated cells. }\end{array}$ & Hybrid & Fig. S9 & $5 A$ \\
\hline $\begin{array}{l}\text { ERK and AKT inhibition induced cell death } \\
\text { mechanisms are predominantly BIM dependent, not } \\
\text { BAD dependent. }\end{array}$ & Hybrid & $\begin{array}{l}\text { Fig. } \\
\text { S10A }\end{array}$ & $5 \mathrm{C}$ \\
\hline $\begin{array}{l}\text { EGF and insulin cooperatively induce cell cycle entry, } \\
\text { with insulin inducing very little cell cycle entry alone. }\end{array}$ & Hybrid & $\begin{array}{l}\text { Fig. } \\
\text { S10B }\end{array}$ & $6 \mathrm{~B}$ \\
\hline $\begin{array}{l}\text { Activation of both ERK and AKT pathways are } \\
\text { required for robust cell cycle entry. Time averaged } \\
\text { ppERK and ppAKT levels correlate with Cyclin D } \\
\text { levels. }\end{array}$ & Deterministic & Fig. S11 & $6 \mathrm{E}$ \\
\hline $\begin{array}{l}\text { The number of ribosomes within the cell doubles } \\
\text { within } 24 \text { hours. }\end{array}$ & Deterministic & $\begin{array}{l}\text { Fig. } \\
\text { S8B }\end{array}$ & S2D \\
\hline
\end{tabular}

SPARCED model simulation

224 Before presenting particular unit test applications, we wanted to provide an overview of model 225 simulation. We built a Jupyter notebook called RunModel. ipynb (Supp. File 11) to simulate the 
SPARCED model (Fig. 2). This notebook requires the model SBML (from CreateModel . ipynb,

227 Fig. 2A), along with the simulation duration (th), the ligand concentrations (if desired), the name

228 for the output files, and whether the simulation should be deterministic only or hybrid mode (flagD).

229 The rest of the notebook imports necessary packages and model files and runs the simulation 230 (Fig. 2B).

231 As mentioned, the SPARCED model consists of two modules: deterministic and stochastic.

232 The SBML file forms the basis of the deterministic module whereas the stochastic module 233 describes gene states (active/inactive) and mRNA birth/death events for the genes (Fig. 2C).

234 When run in the hybrid simulation mode, the deterministic and stochastic modules exchange 235 information every 30 simulated seconds (Fig. 2D). The current levels of select protein states can 236 induce changes in gene activation/deactivation or mRNA transcription/decay rates. The newly 237 updated mRNA copy numbers change nascent protein translation rates in the deterministic 238 module (Fig. 2D). When run deterministically, the model does not stochastically sample gene 239 activation or mRNA transcription events, and such simulations correspond to an average cell 240 state.

When the RunModel . ipynb notebook is run multiple times in hybrid mode, different single-

242 cell responses are simulated (Fig. 2E). For instance, the activation and phosphorylation of ERK

243 (Fig. 2E left, red lines) and AKT (Fig. 2E right, blue lines) proteins in response to growth factor 244 treatment will show variability across three example cells. Although the amplitude of initial 245 response is similar for all three cells, the longer-term responses are quite different. Our previous 246 analyses showed that such single-cell heterogeneity in the initial concentrations of these proteins 247 could help predict cellular fate, namely cell division (40). These jupyter notebooks provide a 248 simple interface to interact with the SPARCED model. 
Figure 2. SPARCED-jupyter enables single-cell response simulations using Jupyter Notebooks. (A) The model creation notebook processes the user defined input files and converts them into the model SMBL file. The model (SBML file) is compiled for simulations using the AMICI python package. (B) When a model is generated for a new cellular context (using new omics input data), the model creation step is followed by an initialization step to adjust protein translation rate constants and cell death/DNA damage related parameters. (C) The model simulation starts with specifying and importing the SPARCED model SBML (see panel A). The user defines the model file name and the sets four additional parameters: (i) The flag ( 1 or 0$)$ to specify if the model should run in deterministic or in hybrid mode (see D and E), respectively. (ii) The time duration in hours for which the model should run. (iii) The vector of ligand concentrations (in nM) to stimulate the cells. (iv) The output file name. Next, the species initial conditions are, by default, read-in from the "Species" input file. Then, the model file is imported, and the model is simulated according to the specified input. The model outputs three matrices of species concentrations over time at every 30 seconds, the activation states of genes over time (every 30 seconds), and the time points of simulation in seconds. The two former matrices are saved using the user define file name (iv). (D) The model is simulated iteratively for each 30 seconds, where the current species concentrations are inputs for the gene expression module, which then outputs new mRNA levels to update the SBML model states. The model is then run for another 30 seconds, until the total simulation time reaches the user input (th) or until the cell dies. The cell death is decided based on cleaved-PARP levels surpassing the PARP levels. (E) In the gene expression module, in hybrid mode, the model randomly decides which genes become active or inactive, and which mRNAs are transcribed or degraded. This SGEmodule.py script is called every 30 seconds with updated species concentrations, simulated using the models SBML with $\mathrm{AMICI}$ package. (F) When the model is hybrid-simulated three times, the different cell responses are observed. Shown are serum-starved average cells stimulated with full growth media for 24 hours. Plotted are free ppERK and ppAKT species concentrations (nM).

SPARCED model Unit Testing: deterministic

We first tested agreement between deterministic Bouhaddou2018 and SPARCED model simulations. The SPARCED model simulations recapitulated the response of an average (deterministic) cell under different stimulation conditions, to within simulation error (Fig. 3A). As an example, we highlight SPARCED model simulations of the cell response (MCF10A cells) to treatment with EGF alone or EGF+insulin (Figs. 3B and S12). Treating growth factor and serumstarved MCF10A cells with EGF and insulin induces activation of ERK, AKT, and their downstream signaling partners, which together influence cell proliferation $(40,70,71)$. The stimulation increases and prolongs AKT and its downstream EIF4EBP1 phosphorylation (Fig. 3B). 
The simulation results from the Bouhaddou2018 model (the solid lines) and SPARCED model (circles) are indistinguishable. The SPARCED-nf implementation, which runs on a highperformance cloud computing infrastructure, similarly reproduces the original simulation data (Fig. 3B, triangles). These results, together with all other deterministic tests in Table 1 (Figs. S3-8 and 11), confirm that the SPARCED model recapitulates the Bouhaddou2018 model simulations and unit tests in deterministic settings. Thus, the simple input file structure combined with automatic model generation is equivalent to the prior MATLAB instantiation in this regard.

Fig 3. SPARCED model recapitulates experimental observations and deterministic/hybrid (deterministic + stochastic) simulation results of the Bouhaddou2018 model. (A) Summary of comparisons of SPARCED model deterministic simulations to Bouhaddou2018 model simulations. The area under the curve (AUC) values of each simulation (see Fig. S12) are calculated and plotted for the two model results. (B) Simulation results from Bouhaddou2018 model (line) and SPARCED-nf model (triangle) run on Kubernetes cluster workflow are the same as SPARCED model (circle) results. Comparisons of selected panels from $(A)$ are shown only. (C) Experimental and stochastic simulation results from Bouhaddou 2018 model are reproduced by SPARCED model simulations. Each dot is a different condition, explained in Fig. S13A. Error bars show experimental or simulation standard error of the mean. Simulations are of at least 100 cells, and three independent experimental observations where applicable. (D) Stochastic simulation of 100 cells recapture protein level trajectories (active p53, Cyclin A, and cPARP) from older model qualitatively. Panels with blue background are SPARCED simulations and white background panels are from Bouhaddou2018 model. 100 stochastic cells are stimulated with EGF+Insulin for 72 hours before Etoposide treatment for another 72 hours. Etoposide is stimulated also with EGF+Insulin. Results for Etoposide treatment without prior growth factor stimulations are shown in Fig. S13B. (E) Quantification of results in (D) shows that SPARCED model simulations coincide with earlier observations in percentage of death induced by etoposide treatment. See Fig. S13C for the effect of no growth factor stimulation before Etoposide treatment. Bars represent mean \pm s.e.m.

317 Next, we evaluated the SPARCED model for stochastic unit tests in single cell simulations. Each

318 single simulated cell has different initial protein levels and dynamics due to stochastic gene 319 expression, and thus may respond differently to the same treatment. The SPARCED model 320 stochastic simulations closely matched Bouhaddou2018 model results, to within simulation error 321 (Figs. 3C and S13A). As an example, we highlight here how single cells respond stochastically to 
322 DNA damage. Etoposide, a chemotherapy drug, induces double- and single-stranded DNA 323 damage, causes cell cycle arrest, and leads to cell death (72). Experiments showed that in the 324 absence of EGF and insulin (to promote cell cycle exit), there is minimal etoposide-induced cell 325 death (Fig. S13B-C) (40). However, in the presence of EGF and insulin (to drive cell cycle 326 progression), etoposide-induced cell death increases over time and reaches around $60 \%$ of the 327 cells (Fig. 3D-E). Simulating etoposide treatment of cycling cells induces robust p53 pulses, 328 disruption of Cyclin A dynamics/cell cycle arrest (Fig. 3D), and more cell death relative to non329 cycling cells (Fig. 3E). These results closely match experimental data and Bouhaddau2018 330 simulations. We conclude that SPARCED model captures DNA damage induced single-cell death 331 percentage and cell cycle state-dependent effect of etoposide. The SPARCED model also passed all other stochastic/hybrid unit tests (Table 1, Figs. S2, 6,7,9, and 10).

Different cell types have different mRNA and protein expression levels, and many mechanistic models assume that it is different expression levels that drive different phenotypes, as opposed to changes in biochemical rate constants. These constants are based on biophysical events like binding, which are based on molecular structures. Here, we tested the ability of the SPARCED model to be re-"initialized" to study different cell types by changing initial levels of total proteins and mRNAs without changing the model topology. Thus, we introduced a protocol to enable SPARCED model context change (Fig. S14A and Supp. Files 12 and 13). In short, OmicsData,

341 Species, and Ratelaws input files are updated with new cell line information, including mRNA

342 levels, protein/species levels, and constitutive translation rate constants. Then, the new model is created by running the "createModel" Jupyter notebook or by submitting a new SPARCED-nf 344 job.

345 The re-calibration step for context change followed in Bouhaddou2018 model was called 346 Initialization, where protein-specific translation rates and key parameters important for cell 
decision making are estimated to ensure agreement with new omics datasets and expected phenotypic behavior with respect to proliferation and apoptosis. Here we also provide a new, python-based version of the Initialization procedure for SPARCED models (see Computational Methods), where the outputs are species concentrations and rate parameter values updated in a new SBML file. Here, to test the drug combination response differences in different cell lines, we changed SPARCED model context (i.e. parameter values and species concentrations) by initializing the model to the U87 glioma cell line. Following the protocol outlined in Fig. S14A, we replaced MCF10A cell line values in the input files with values from U87 cell line data.

U87 cells are PTEN-deficient and more sensitive to AKT inhibition compared to MCF10A cells (40). Both cell lines show minimal sensitivity to MEK inhibition alone and AKT \& MEK inhibitors are both needed to kill MCF10A cells. In contrast, AKT inhibition alone is sufficient to kill U87 cells. To simulate the U87 cell response to AKT and MEK inhibitors, we first updated the OmicsData input file (Supp. File 1) using U87 mRNAseq data from Bouhaddou2018 model (Supp. File 14). Here, we did not have U87 cell line proteomic data and estimated the initial total protein levels using the new mRNA levels and gene-level mRNA/protein ratios from MCF10A data (Supp. File 15). We set PTEN translation rate to zero and set values of rate parameters dictated by Initialization in the Ratelaws input file (Fig. S14B and Supp. File 12). Additionally, we provide an improved Python based initializer InitializeModel. ipynb notebook (Supp. Files 16 and 17), which re-creates (Fig. S15) the un-stimulated steady-state initial conditions for species and adjusts translation rate constants using cell-line specific initialization input file (Supp. File 18). We also updated the species initial conditions in Species input file using steady-state values for U87 cells from the Bouhaddou2018 model (Supp. File 19). We created a new model SBML file (SPARCED_U87) using the updated input files. SPARCED_U87 model simulations of response to MEK and AKT inhibitors reproduced the Bouhaddou2018 model results and experimental 
371 observations (Fig. S14C). We conclude that changing model context by changing input files

372 accomplishes the goal of easy model alteration to study of different cell types.

373 When the cellular context (omics input data) for the SPARCED model is changed, all 374 appropriate Unit Tests should pass. We expect that addition and alteration of the list provided

375 (Table 1) will accommodate increasingly different prior knowledge about the new context. 376 Examples of such information include cell line mutations, growth condition differences, or tumor 377 cell behavior.

IIlustrating easy model expansion by application to the IFN $\gamma$ pathway: SPARCED-I

379 The SPARCED model reproduces original model results and experimental observations, but how 380 can we use the new simple model expansion capabilities? Here, we focused on experimental observations that interferon-gamma (IFN $\gamma$ ) inhibits MCF10A cell proliferation. Specifically, as part of a much larger LINCS consortium effort to deeply profile the MCF10A cell line dynamic response to perturbations (synapse.org/\#!Synapse:syn12526172), we observed that IFN $\gamma$ inhibits EGFinduced cell proliferation (Fig. 4A). We wanted to use the SPARCED model expansion functionality to evaluate the suitability of candidate mechanisms by which IFN $\gamma$ might inhibit proliferation.

Fig 4. SPARCED model is enlarged to include interferon-gamma (IFN $\gamma$ ) signaling pathway. (A) Experiments showed that IFN $\gamma$ treatment decreases cell proliferation induced by EGF alone. Fraction of EdU positive cells at 48 hours after EGF alone or EGF+IFN $\gamma$ treatment are calculated. Bars represent mean \pm s.e.m of three independent biological replicates. Significance tested using two-sided two-sample t-test, where * indicates p-value $<0.05$, ${ }^{* * *}$ indicates $p$-value $<0.001$. (B) Yamada2003 model schematic of IFN $\gamma$ pathway added into SPARCED model. (C) Overview of the added IFN $\gamma$-IFNGR pathway in relation to the Bouhaddou2018 model pathways. The "mech1 and mech2" links are candidate mechanisms tested in the next section. (D) Simulations of the Yamada2003 model, SPARCED-I model, and SPARCED-I model with MCF10A context show qualitative and quantitative agreements. The Yamada2003 model results (blue diamonds) are obtained by running the model file in COPASI (61). (E) 100 stochastic cell simulations (area plots) and the deterministic (dashed-black lines) simulation of $10 \mathrm{nM}$ IFN $\gamma$ stimulation are shown. 400 Colored dark lines represent median cell trajectories, dark and light-colored regions represent $70^{\text {th }}$ 
401

402

403

404

405

406

407

408

409

410

411

412

413

414

415

416

417

418

419

420

421

422

423

424

and $95^{\text {th }}$ quantiles, respectively. The right-most plot shows mRNA count of SOCS1 in each cell, colored differently.

We thus merged a model for interferon-gamma receptor (IFNGR) signaling into SPARCED, creating the model variant SPARCED-I. The newly added Yamada2003 model (73) captures how IFN $\gamma$ binds to pre-JAK-bound receptors, inducing dimerization. The ligand-bound receptor homodimers are activated by JAK, which also phosphorylates STAT1 when bound to the active receptor complex. Activated STAT1 then dimerizes and translocates to the nucleus, inducing transcription of SOCS1. SOCS1 mRNA is exported to the cytoplasm and translated into SOCS1 protein. SOCS1 protein binds to and inhibits activated receptor homodimers. In this model, there are three phosphatases (SHP2, PPX, and PPN) acting on multiple species.

Following the model alteration protocol in Fig. S14A and Supp. File 13, we added 34 new species, 8 corresponding genes, and $\sim 70$ reactions (Fig. 4B) based on the Yamada2003 model (73). New genes (IFNG, IFNGR, JAK2, STAT1, SHP2, SOCS1, PPN, PPX) corresponding to the proteins in the model are added as new rows and mRNA levels are inserted into the OmicsData input file. Gene copy numbers are taken as two (40). The genes are also added as new rows to the GeneReg file (Supp. File 20). In the Yamada2003 model, activated nuclear STAT1 dimers $\left(\mathrm{STAT1}^{*} \mathrm{Dn}\right)$ induce SOCS1 mRNA transcription and this is captured by adding a new column in the Genereg input file, with the only non-zero element at the STAT1*Dn and SOCS1 gene intersection. Next, each protein, protein complex, and mRNA species are inserted into the Species and StoichiometricMatrix input files as new rows. Each new reaction is inserted into the Ratelaws input files as rows, and into the StoichiometricMatrix input file as new columns. This expansion brought the total number of species of SPARCED-I model to 954, and the number of reactions to 2540 (Fig.4C, Supp. File 21). 
426 The SPARCED-I model, with parameter values from the Yamada2003 model, should reproduce

427 the original results exactly, which we verified (Fig. 4D, red lines \& diamonds, respectively). We

428 then modified the mRNA, protein, and compartment volume values to that of MCF10A cell context

429 (data from (40)). However, the MCF10A data (Supp. File 22) had missing values for IFNGR and

430 (arbitrary) phosphatase species PPN and PPX. So, we initialized the concentration of IFNGR as

431 half of JAK2 concentration (the receptor is typically rate limiting (73)). The concentrations of PPN

432 and PPX phosphatases were equal to half of SHP2 concentration in Yamada2003 model, so we

updated their values to half of SHP2 concentration in MCF10A cells. In addition to the reactions

434 from the Yamada2003 model, we added new translation (for the new eight genes) and degradation (for all new species) reactions into the model. The rate constants of these extra reactions are initially assumed to be equal to the average of corresponding reactions of

SPARCED model genes. Starting from these parameter values, the SPARCED-I model showed unrealistic (ultrafast) receptor activation and STAT1 phosphorylation/nuclear transport rates.

439 Therefore, we varied six parameters that have high impact on STAT1 activation dynamics (see Methods) to approximate the timing (within the first hour) of STAT1*Dn pulses reported in

441 Yamada2003 model (Fig. 4D) and others (74,75). Changing rate constants in such a manner 442 accounts for the entangled effects of unmodeled cellular context and mechanisms. Tuning these 443 parameters produced expected pulsing times and response behavior of STAT1*Dn, SOCS1, and 444 SOCS1 mRNA levels (Fig. 4D black lines). The final values are updated in the SPARCED-I model 445 file (Supp. File 23).

446 A key feature of SPARCED-I is its ability to simulate single cell behavior and the above447 observed reduction in proliferation induced by IFN $\gamma$ is inherently a single cell property. As a unit 448 test, we simulated 100 single cell trajectories (Fig. 4E) of SPARCED-I model and concluded that SPARCED-I model recapitulates observations from earlier models and passes all unit tests. 


\section{SPARCED-I model variant analysis: hypotheses testing}

451 Next, we wanted to use the expanded model to help us interpret the experimental observations.

452 How does IFNy inhibit EGF-induced cell proliferation? The SPARCED model captures regulation of cell proliferation via the ERK and AKT pathways. Growth-inducing ligands, like EGF, bind to and activate receptor tyrosine kinases (RTKs), which in turn leads to upregulation of AKT and ERK phosphorylation. The two pathways together induce upregulation of cyclin D through cJUN, cFOS, and cMYC activities $(40,70,76)$.

In the literature, there are different mechanisms by which IFN $\gamma$ was suggested to play a role in cell proliferation (77-80). The SPARCED-I model enabled us to evaluate these hypotheses for consistency with experimental observations (Fig. 4A), where the simulation steps are matched to the experimental setup (Fig. 5A). The first proposed mechanism (mech 1, Fig. 5B) was based on reports that activated STAT1 can induce p21 (cell cycle inhibitor) expression $(77,81)$. This mechanism was modeled (Supp. File 24) by modifying the GeneReg input file (Supp. File 21), had p21 transcriptional activation by $\mathrm{p} 53$, and the rate constant for transcriptional activation of p21 by STAT1 was taken to be equal to the parameter values from the p53 mechanism.

Fig 5. Study of SPARCED-I model enabled us to propose how IFN $\gamma$ treatment decreases cell proliferation induced by EGF. (A) Experimental setup and simulation workflow for SPARCED-I model variant analysis. See Methods. (B) Candidate mechanism schematic 1: activated STAT1 induces p21 transcription. (C) Candidate mechanism schematic 2: SOCS1 sequesters activated ligand-receptor complexes. (D) Simulation of 100 stochastic cells for SPARCED-I-mech1 and (E) SPARCED-I-mech2 models showed that SOCS1 sequestration of activated receptor complexes can better explain the IFN $\gamma$ effect on cell proliferation inhibition, compared to experiments shown in Fig. 4A. The bar plots show the total number Cyclin A peaks calculated at specified time points for the starting 100 cells. (F) Normalized p21 levels do not show a significant change when IFN $\gamma$ is included in addition to the EGF. (G) Normalized ppAKT levels show a significant decrease after IFN $\gamma$ treatment, when EGF+IFN $\gamma$ Mechanism 2 simulations are compared to EGF alone case. RPPA data are shown in black error lines, from three independent replicates. Colored dark lines represent median cell trajectories from 
481

482

483

484

485

486

487

488

489

490

491

492

493

494

495

496

497

498

499

500

501

502

503

504

505

Significance tested using two-sided two-sample t-test, where * indicates $p$-value $<0.05$, *** indicates p-value < 0.001. (F-G) Data from synapse.org/\#!Synapse:syn12526172.

The second mechanism involves the negative regulator of IFN $\gamma$ signaling, SOCS1. SOCS1 protein has different binding domains, including $\mathrm{SH} 2$ domains $(82,83)$. $\mathrm{SH} 2$ domains bind to phosphorylated tyrosine residues on other proteins $(84,85)$. It is proposed that SOCS1 not only binds to activated IFN $\gamma$ receptors, but also to many other activated receptor complexes with free phosphorylated tyrosine residues (mech 2, Fig. 5C) $(83,86,87)$. Thus, IFN $\gamma$-induced SOCS1 protein can bind to growth factor-activated receptor complexes (or the so-called signaling competent dimers - pSCD) and prevent further downstream signaling by sequestration. This mechanism was modeled by adding SOCS1 binding to activated receptor complexes (pSCDs) reactions in the Ratelaws input file. The SPARCED-I mech2 model contained 1302 species (348 new) and 3584 reactions (1044 new) (Supp. File 25). GRB2 proteins also contain SH2 domains that bind to tyrosine phosphorylated receptors (pSCDs) and the rate constants of SOCS1 interaction with all these complexes are taken as the average of such parameters of GRB2 complexes.

Before evaluating these model variants, the initial conditions must be set. The SPARCED-I model initial conditions are based on serum and growth factor starved MCF10A cells. However, the experiments with IFN $\gamma$ (Fig. 4A) were done in media with horse serum. Horse serum upregulates ppAKT levels by four-fold (Figs. S16 and S17), possibly thorough IGF/IGF1R pathway (88-90). Including 0.02 nM IGF meets this basal activity increase constraint, and therefore is included in simulations prior to and during simulated EGF and IFN $\gamma$ treatments (see Methods and Figs. S16-19).

After creating the SPARCED-I Mechanism 1 and 2 variants and defining the simulation conditions, we stochastically simulated both models with IGF treatment for 24 hours for 100 
different single cells to provide a baseline. Then, either EGF or EGF + IFN $\gamma$ are added for an

507 additional 48 hours for each cell (Fig. 5A). As a simulation metric for cell proliferation, we counted

508 and summed the number of cyclin A peaks in each of the 100 starting cells for the 48 hours (Fig.

$5095 \mathrm{D}$ and E-orange and blue bars). Mechanism 2 leads to a significant decrease in the number of 510 cell cycles in IFN $\gamma$ treated cells, whereas Mechanism 1 did not cause a change in EGF and 511 EGF+IFN $\gamma$ conditions.

Exploring further, we saw that Mechanism 1 did not lead to significant increases in p21 protein levels (area plots in Fig. 5F). While it is unexpected that the model predicted no significant increase in p21 levels despite a clearly added mechanism, if the parameter value for STAT1 induction of p21 transcription is changed enough (see Supplemental Equation 12 in (40)), simulations do indeed show IFN $\gamma$ treatment induces p21 (Fig. S20). However, experimental RPPA data from the LINCS Consortium confirm that total p21 levels do not significantly change over the course of 24 hours in EGF+IFN $\gamma$ treated cells compared to EGF alone stimulation (error bars in Fig. 5F, synapse.org/\#!Synapse:syn12526172). The experimental 48 hour time point showed elevated p21 levels, which is different from simulations. We conclude that the original parameter choices are appropriate and that the putative p21 mechanism is therefore unlikely. simulated cells enter the cell cycle (Fig. 5E). We saw a decrease in MAPK activation in IFN $\gamma$ 524 treated cells, supported by experimental data, but in both Mechanisms 1 and 2 (Fig. S21). Then, 525 looking at AKT responses, we saw lower in silico AKT phosphorylation level in IFN $\gamma$ treated cells of only Mechanism 2, compared to only EGF stimulated cells (Fig. 5G area plots). The greatest decrease occurs at around one hour after stimulation and stays decreased for 48 hours. We compared this simulation result to experimental RPPA data (synapse.org/\#!Synapse:syn12526172), which also show decreased ppAKT levels at 1-4 hours post-ligand treatment (Fig. 5G error bars). The variability in the 8 hour measurement was large, 
531 and the 24 hour measurement showed still reduced ppAKT. By 48 hours, ppAKT is upregulated

532 in the experimental data, which the model simulations do not account for. Is the somewhat subtle

533 decrease in AKT activity for $\sim 1$ day enough to prevent cell cycle entry in MCF10A cells?

534 Bouhaddou et al. showed that a slight change in time integrated AKT dynamics can tilt the cell

535 cycle progression decisions significantly (see Fig. S11 and (40) for further details) and here we

536 saw ppAKT levels show a statistically significant decrease after IFN $\gamma$ treatment, which may explain

537 fewer proliferative cells. Taken together, these results suggest that: (1) the SPARCED model

538 formalism can be used for simple implementation of large-scale mechanistic model-based

539 hypotheses testing, (2) STAT1 induction of p21 expression levels is an unlikely mechanism in this

540 MCF10A cell context, (3) crosstalk between IFN $\gamma$ and EGF pathways may occur through SOCS1

541 sequestration of activated receptor domains, and (4) SOCS1-induced inhibition of AKT activation

542 may contribute to proliferation suppression. 
544

545

546

547

548

549

550

551

552

553

554

\section{DISCUSSION}

Here, we have re-created one of the largest mechanistic models in the literature, using our new python-based creation and simulation pipeline. Enabling large-scale, single-cell modeling as a data integration tool, our pipeline is based on structured and easy to modify input text files and uses Jupyter notebooks or scripts (for scaled cloud-computing) to create and simulate model files. It also enables easier model alteration (species/rate law or parameter value changes), omics data integration, and model variant vs. hypotheses testing. Our exemplar model, called SPARCED, is available online on GitHub (github.com/birtwistlelab/SPARCED). SPARCED can serve as a basis for creating context-specific (personalized) model variants, studying virtual cell population responses, and as a building block towards whole-cell-scale models.

First, we showcased the use of SPARCED model by changing the cellular context of the model from MCF10A breast epithelial cells to U87 glioblastoma cells, by only replacing parameter values in three/four input files. Although we used previously calculated values from the Bouhaddou2018 model to show reproducibility of the subsequent analyses, it is notable that SPARCED pipeline correctly creates and simulates a large-scale mechanistic model file only from an altered set of text-based input files. Additionally, we provided a new version of the Initialization script (Supp. File 16) that utilizes another cell-line specific input file (Supp. File 18) to calibrate the model initial conditions. The initialization allows distribution of total protein and mRNA level omics data across all model species and estimates data-driven, cell-line specific translation rate constants. As a customizable set of steps, the initialization sustains user defined phenotypic responses, like cells not going into apoptosis or cell cycle without growth stimulation. Importantly, the procedure accommodates mRNA input alone (without proteomics data) and calculates total protein levels using gene-level mRNA-to-protein ratios from the default MCF10A values. The output of the initialization procedure are species concentrations and parameter values deposited into a new SBML file, which is exported with a new name and re-compiled using AMICI. 
Secondly, we investigated two candidate mechanisms to explain how IFN $\gamma$ can inhibit EGF-

570 induced cell proliferation. By creating and testing two mechanisms (by only changing the input

571 files), we hypothesized that IFN $\gamma$-induced SOCS1 sequestration of activated receptors is a more

572 likely mechanism than IFN $\gamma$ induction of cell cycle inhibitor protein p21. Besides these two

573 mechanisms tested, there are others in the literature, like the positive feedback of STAT1 inducing

574 STAT1 and IRF1 transcription (78), or the inhibition of Bcl-2 by STAT1 (77). However, here, we

575 only focused on demonstrating the capabilities of SPARCED pipeline to easily create and test

576 model variants to help explain one of our experimental observations, where testing all possible

577 mechanisms was out of our scope.

Many existing big models are constructed in complicated and hard-coded ways and are not available in standard modeling formats, like SBML. For instance, the Bouhaddou2018 model we used as our starting point was custom coded in MATLAB with tens of different script files with upon structured and annotated input files, by using open-source packages, and by being available 587 publicly on GitHub.

One key advantage of the SPARCED model format is its potential compatibility with RBM. 589 The reactions and species created by RBM software can be incorporated (manually or programmatically) into the SPARCED model input files. Although existing RBM software can export models in SBML format and enable multiple features, the SPARCED models enable single rate parameter changes and inclusion/exclusion of individual rate laws at the input file level. Then, 
594

595

596

597

598

599

600

601

602

603

604

scanning. One main goal of the $\mathrm{AMICl}$ package (91) is enabling large-scale parameter estimation, and our choice to use this package was to enable such future endeavors when needed. Combining this idea to test consistency across multiple datasets, users can search for best-fit models or pinpoint discrepant datasets given the model topology (28).

Another advantage of the SPARCED model will be its ability to integrate multiple omics datasets into a large-scale mechanistic model, creating a "personalized" model variant reflective of another cellular or patient context $(26,92)$. With the Initialization procedure linked to our pipeline, users can incorporate mRNA, copy number variation (CNV), and even proteomics data from established databases like CCLE (93), TCGA (94-96), HPA (97-99), and Cellosaurus (100) into the input files programmatically (Fig. S14) and test changing the initial conditions of the model using the same network structure (Fig. 4).

The SPARCED model encodes intrinsic stochasticity of total protein levels and mRNA numbers in its hybrid simulation mode, making it unique (together with the Bouhaddou2018 model) to offer stochastic as well as deterministic simulation settings. Although other tools such as COPASI offer hybrid (deterministic + stochastic) simulation settings, our approach always treats the gene expression module as stochastic, and the events modelled as Poisson processes. COPASI uses next-reaction-method (101) for the part it determines as stochastic based on molecule numbers of the interacting species. However, as the developers stated, such implementations tend to be inefficient and take prolonged simulation wall-times. The single-cell capability of SPARCED allows one to capture some important aspects of cell line and tumor heterogeneity compared to an average cell condition (the way many mechanistic models are built). Users can leverage this feature to simulate virtual populations and study a cell population response to drug treatment, which is often a single-cell readout as are most cellular phenotypes. However, such simulation settings require larger computational resources and thus model compatibility for high performance computing environments. The SPARCED model is built to be 
619

620

621

622

623

624

625

626

627

628

629

630

631

632

633

634

635

636

637

638

639

640

641

642

643

compatible with cloud computing, where it can be used to simulate thousands of single cells with single job execution (see Methods).

There are, of course, remaining challenges. One such task is to explore simulations of spatially aware single cells. Currently, the SPARCED model captures intrinsic heterogeneity of cells (by having stochastic gene switching and mRNA birth/death events) but these cells cannot "talk" to each other. In the future, by having scenarios where spatial orientation of cells are recorded and the secreted or stimulated molecules are shared between them, we can better capture tissue microenvironment and heterogenous pharmacokinetics $(102,103)$. Related to this first task, the second challenge is to create and simulate scenarios with multiple cell types (i.e. models trained on data from different subtypes of cells) or defining events to capture differentiation of cells. For example, one may be able to use single cell RNAseq data to train SPARCED-like models to enable tissue-level simulations with the critical cell types in the proper geometric locations. This overall vision would enable spatially aware, single-cell level, large-scale mechanistic models trained on individual patient data for in silico drug screening. The pipeline presented here is an important step towards this goal.

Another challenge to achieve using large-scale models is a whole-cell level mechanistic model for mammalian cells $(56,104)$. With our approach, the SPARCED model can be enlarged using other small-scale models for pathways and mechanisms not currently included in the model. By utilizing the unit testing approach, one can then verify the model performance and get larger, more comprehensive models. The open-source framework presented here increasingly facilitates community contribution for model context-change and parameter tuning based on new experimental conditions.

Our introduced method of large-scale mechanistic model construction, and the SPARCED model as a basis, will enable researchers to more easily create and manipulate new model versions, test different mechanisms of action to interpret experimental observations, and change 
644 the model's cellular context. The models created by our pipeline can incorporate multiple (omics)

645 datasets, providing non-"black-box" data integration and modeling. These SPARCED models

646 additionally provide single-cell level simulations, compatibility with cloud computing, and human-

647 interpretable \& annotated model files in SBML format. The SPARCED model now can more easily

648 be re-used as one of the largest mammalian-cell mechanistic model in the literature and serves

649 a primer role in creation of context-specific, hypotheses testing, and expandable models. In

650 conclusion, the SPARCED model format contributes towards important foundations of reusable

651 big models, paving the way towards personalized mechanistic models for data integration.

652 


\section{MATERIALS AND METHODS}

654 Computational Methods

\section{The Bouhaddou2018 model}

656 The Bouhaddou2018 model (Fig. 1A) is one of the largest single-cell mechanistic models for 657 mammalian cell signaling regulating proliferation and death. The first version of the model used 658 as a test case in this work was written in MATLAB (The MathWorks, Inc.) (40). The model is a 659 hybrid of deterministic and stochastic modules. The deterministic module describes the 660 concentration dynamics of 774 proteins, protein complexes, and post-translationally modified species through 2449 reactions using the Sundials CVODEs package for simulation (105). The stochastic module describes gene state (active/inactive) and mRNA birth/death dynamics for 141 genes. The deterministic and stochastic modules exchange information every 30 simulated seconds. In short, the current levels of select protein states can induce changes in gene activation/deactivation and/or mRNA transcription/decay rates. The newly updated mRNA copy numbers change nascent protein translation rates in the deterministic module. See (40) for further

667 details.

\section{The SPARCED model}

669 We converted the Bouhaddou2018 model into a Python + SBML (59) format (Fig. 1B). The deterministic module is ultimately encoded in an SBML file (.xml) whereas the stochastic module

671 is written in Python. A foundational and important feature of this recoding effort is that the SBML

672 file is generated from a small set of simple structured input text files (Fig. 1C) via Python scripts.

673 Introduction of such structured input files and associated Jupyter notebooks enables simple

674 alteration of model structure and/or parameter values, for example turning on/off certain 675 interactions. The input files also enable rigorous annotation of model features using, for example, 676 ENSEMBL (106) and HGNC (107) identifiers, which is seldom done in such mechanistic 677 modeling. 
678

Input files

679 There are six SPARCED model input text files (tab separated values), each with a defined 680 structure as detailed below. The user can change these files to create and compile a new model.

681

682

683

684

685

686

687

688

689

690

691

692

693

694

695

696

697

698

699

700

701

702

(1) OmicsData: This file (Supp. File 1) includes the gene copy number, mRNA copy number, and proteomic data. This input file also contains rate constants for the stochastic module and initialization procedure. Each row of the file corresponds to one gene and the columns are different data types. The first column is gene name (HGNC identifiers), the second column is gene copy number, the third column is mRNA molecule copy number per cell (mpc), the fourth and fifth columns are rate constants of gene inactivation and activation respectively $\left(\mathrm{s}^{-1}\right)$, the sixth column is constitutive transcription rate constants (molecules per second), the seventh column is maximal transcription rate constants (molecules per second), the eighth column is mRNA degradation rate constants $\left(\mathrm{s}^{-1}\right)$, the ninth column is protein copy number $(\mathrm{mpc})$, the tenth column is protein halflife parameters (seconds), and finally the eleventh column is the translation rate constants $\left(\mathrm{s}^{-1}\right)$. These latest set of rate constants are from literature and provided for genes for which our omics input lacked protein level data. All the rate constants are taken from the Bouhaddou2018 model. Users can add new rows to this file, using RNAseq data to estimate mRNA levels for the genes to be added (40). When adding genes (rows) to the model, a reasonable starting point for rate constants (or other values), in the absence of any other data, is to use median values from the genes/parameters currently in the model.

(2) Species: This file (Supp. File 2) contains information about the species in the deterministic module. Each row corresponds to one species (protein, protein complex, post-transcriptionally modified species). Transcripts (in $\mathrm{nM}$ ) are also included in this file because they are regarded as species with updated concentrations in the stochastic module every 30 seconds and are used in translation rate laws. The first column is the species name. Names can be arbitrary so long as they are unique in the model. Importantly, the name list needs to match the first column in the 
StoichiometricMatrix file described below. The second column is the species home

704

705

706

707

708

709

710

711

712

713

714

715

716

717

718

719

720

721

722

723

724

725

726

727

compartment. The home compartment of a species defines its cellular localization. A species can reside in a compartment defined in the compartments input file: currently Cytoplasm, Mitochondria, Nucleus, or Extracellular. The third column is initial condition in nM units, with respect to the home compartment volume. These values are taken from the Bouhaddou2018 model, post-initialization. The fourth column is a comma separated list of ENSEMBL gene identifiers corresponding to gene products present in the species.

(3) Ratelaws: This file (Supp. File 3) has a row for each reaction in the deterministic module.

The first column is the unique (arbitrary) name of each reaction. Currently, we named each reaction based on the related sub-module (e.g. vA1-87 for Apoptosis and vC1-104 for Cell Cycle). The number and order of rows in this file should match the columns in the StoichiometricMatrix input file defined below. The second column in this file contains the home compartments for the reactions. The designated compartments should be one defined in the Compartments input file: currently Cytoplasm, Mitochondria, Nucleus, or Extracellular. The home reaction compartments define the effective search volume for each reaction and is used to rescale concentrations when appropriate. Note that both species and reactions have home compartments defined, where a species can participate in a reaction defined in a different compartment. For instance, the EGF binding to EGFR reaction occurs in extracellular space (volume $V_{e}$ ), where EGF's home compartment is the extracellular space and EGFR's home compartment is cytoplasm $\left(V_{c}\right)$. A volumetric correction for EGFR concentration in this rate law is done by multiplying by the ratio of $\mathrm{V}_{\mathcal{c}} \mathrm{V}_{\mathrm{e}}$. The third column can have either a number or a reaction formula. If it is a number, it means the corresponding reaction is mass-action type, and the number is the rate constant for that reaction in units of $\mathrm{nM}$ and seconds. Note that the reactants and products are defined in the StoichiometricMatrix input file. If the third column is a formula, it means the reaction will follow that rate law, and the next set columns in that row are the values 
of each parameter defined in the formula in the third column, again in units of $\mathrm{nM}$ and seconds.

729 The rate law can include any species name described in the Species input file. The parameter

730 names in the rate law should start with " $k$ " and be unique in that formula. We distinguish multiple

731 parameter names with an underscore and ascending list of numbers (e.g. kA_1, kA_2). During

732 model generation, all parameter names in this file are re-named in an ascending order based on

733 the number of rate laws. The full list of parameter name/value pairs are outputted into a new file

734 (ParamsAll) for user reference.

(4) StoichiometricMatrix: This file (Supp. File 4) defines the reaction stoichiometry, and

therefore the reactants and products of model reactions. The rows correspond to the Species

737 input file and the columns correspond to the rows in the Ratelaws input file. Here, the species

738 and rate law names should match the names defined in Supp. Files 2 and 3. Each element

739 (starting at the second row and second column index) has a stoichiometric coefficient (typically -

$7402,-1,0,1$, or 2$)$, where negative sign indicates reactants, and positive sign implicates products of

741 a reaction.

(5) GeneReg: This file (Supp. File 5) describes transcriptional activation and inhibition

743 interactions, where rows correspond to genes (the same order as the first column of Supp. File

744 1) and columns to species that are defined as activators or repressors of transcriptional activity.

745 The first column is gene name (HGNC format). There are currently seven more columns in this

746 file, each corresponding to one species defined as an activator or a repressor (e.g. p53 induces

747 p21 transcription or AP1 inhibits cFOS transcription). A single value of zero indicates no effect. A

748 non-zero entry in row $\mathrm{i}$ and column $\mathrm{j}$ denotes that species $\mathrm{j}$ regulates gene $\mathrm{i}$ transcription. The

749 non-zero entries have the form " $A$; $B$ ", where " $A$ " is the hill coefficient and " $B$ " is the half-maximal

750 concentration of the species "j" effect. To simplify the input file structure, we use positive values

751 of "A" to denote activation, and negative "A" values to denote inhibition. This file is used by the

752 stochastic module script to update mRNA levels. To add additional transcriptional regulators 
753 (activators or repressors) into the SPARCED model, users should add as many columns as new

754 regulator species and populate the columns with corresponding rate constants.

755 (6) Compartments: This file (Supp. File 6) contains the names of compartments in the model 756 (first column), the volume of the compartment in liters (second column), and the corresponding 757 GO-term of the compartment (third column). The compartment names should match the compartment names listed in Species and Ratelaws input files.

(7) Observables: This file (Supp. File 7) contains information about model observables. Each observable corresponds to the compartmental-volume-corrected summation of all formats of a protein. There are 102 observables defined (columns) for the model species (rows) in this file.

762 The entries are either 1 (the species in the row is part of the observable in the column) or 0 763 (otherwise). The "createModel" Jupyter notebook (Supp. File 8) uses this file to define an observables variable as an input for the AMICI model compiler.

(8) Initializer (Optional): This file (Supp. File 16) contains information used for model 766 initialization. Species concentrations (columns 1-2), mRNA level adjustments (columns 3-4), 767 parameter values (columns 5-7), observables to exclude from translation rate adjustments 768 (column 8), and single parameter scan range (columns 9-11) are populated for each step of 769 initialization. The steps used here are shown to work to get a good starting point for serum starved

770 MCF10A cells, which do not undergo apoptosis or enter cell cycle without growth factor 771 stimulation, in deterministic simulation mode.

\section{Dependencies}

773 (1) Docker: All model dependencies and runtime environments are Dockerized into a 774 downloadable image for self-contained model execution. To run the SPARCED model using 775 Jupyter notebooks (SPARCED-jupyter), Docker must be installed. Then, by downloading the 776 docker image, built on the Ubuntu-18.04 operating system with python3 installed 
777 (hub.docker.com/repository/docker/birtwistlelab/sparced-notebook), users can run the Jupyter

778 notebooks defined below in any web-browser within the docker container. The Docker image

779 includes system utilities required for the simulation package AMICI $(67,69)$.

780

781

782

783

784

785

786

787

788

789

790

791

792

793

794

795

\section{Jupyter notebook 1: model creation}

The input files described above are processed by the "createModel" Jupyter notebook (68) (Supp. File 8 and converted into an Antimony (63) text file (Fig. 1B and Supp. File 9). This intermediate step and file provide an additional means to model input, fine-tuning, and alteration for experienced users. It can be explored via any text editor and it lists all elements of the model: species, rate laws, parameters, compartments, and corresponding values (Supp. File 9). This text file is then converted into an SBML (.xml) file, using libantimony in the same script. The Antimony format does not, to our knowledge, support addition of structured annotations, so the annotations (species and compartments) are added to the newly created SBML file using libsbml and the model input files (Supp. Files 2 and 6). Finally, the annotated SPARCED model file $(. x \mathrm{ml})$ is generated (Supp. File 10).

\section{Deterministic module}

The model SBML file forms the basis of the deterministic module. When run deterministically, the model does not account for stochastic gene switching and mRNA transcription events (see next section). The default parameters and concentrations of the SPARCED model correspond to an average, serum-starved cell state in deterministic mode.

\section{Stochastic module}

In addition to the deterministic module, the SPARCED model includes a stochastic module. The stochastic module describes gene states (active/inactive) and mRNA birth/death events for genes (currently 141 of them). The deterministic and stochastic modules exchange information every 30 simulated seconds. The current levels of select protein states can induce changes in gene activation/deactivation and/or mRNA transcription/decay rates. The newly updated mRNA copy 
802

803

804

805

806

807

808

809

810

811

812

813

814

815

816

817

818

819

820

821

822

823

824

825

numbers change nascent protein translation rates in the deterministic module. See (40) for further details.

The stochastic module constitutes two short Python scripts. At the start of each simulation, one of the scripts (RunPrep.py) reads in the OmicsData input file, processes parameter values, and sets the initial transcript levels and stochastic module rate constants. The second script (SGEmodule.py) uses information from RunPrep.py and species concentrations from the deterministic module to simulate mRNA transcription/degradation and gene activation/inactivation events. One output of the second script is the new concentrations of mRNAs, which is updated in the deterministic module to calculate rates of translation for the next 30 second simulation. The second output is the state of all gene copies (active or inactive).

\section{Jupyter notebook 2: model initialization}

Making use of the created model file and the Initializer input file, total protein abundance data are converted protein and protein complex starting concentrations by adjusting translation rate constants. The "InitializeModel" notebook (Supp. File 16) also verifies that the simulated cells behave as expected in serum-starved state. It is possible to modify the steps of initialization to confer new basal behavior (such as cycling) or a mutational effect (loss of PTEN in U87 cells), as introduced by Initialization protocol in Bouhaddou2018 model. Running this file is optional and recommended only when new models are created for new cell contexts.

\section{Jupyter notebook 3: model simulation}

Supp. File 11 includes an example of simulation setup and input parameters of the SPARCED model. The notebook "runModel" imports a user specified model SBML file and stochastic module scripts to run simulations using the AMICI package $(69,108)$. AMICI is an interface for

Sundials CVODEs solvers that converts SBML files into executable C code for fast simulation. Other required input parameters for model simulation include: a flag to specify if the simulations 
826 are fully deterministic ( $f$ lag $=1$ ) or hybrid (flag=0), the total simulation time in hours (th), input ligand

827 concentrations, and a flag ( 1 or 0$)$ to indicate if results should be exported.

\section{The SPARCED-I model}

SPARCED-I model creation

830 We created a new enlarged version of the SPARCED model called SPARCED-I. We merged

831 Yamada2003 model (73) of interferon-gamma receptor (IFNGR) signaling into SPARCED. The

832 expansion included addition of 34 new species, 8 corresponding genes, and $\sim 70$ reactions. New

833 genes (IFNG, IFNGR, JAK2, STAT1, SHP2, SOCS1, PPN, PPX) corresponding to the proteins in

834 the model are added as new rows and mRNA levels are inserted into the OmicsData input file.

835 Gene copy numbers are taken as two (40). Each new protein, protein complex, and mRNA 836 species are inserted into the Species and StoichiometricMatrix input files as new rows.

837 Each new reaction is inserted into the Ratelaws input files as rows, and into the

838 StoichiometricMatrix input file as new columns. The new genes are added as rows to the

839 GeneReg file. The final SPARCED-I model has 954 species and 2540 reactions.

\section{Parameter estimation of the SPARCED-I model}

After expansion of the SPARCED model with IFNGR pathway and setting the initial species levels from MCF10A cells, SPARCED-I model showed ultrafast receptor and STAT1 activation dynamics inconsistent with biological observations. Thus, we selected six rate constant parameters for calibration based on substantial sensitivity for STAT1 activation dynamics. The parameters calibrated are: (i) STAT1 binding to activated receptor complexes, (ii) nuclear translocation rate of STAT1* dimers, (iii) SOSC1 mRNA translation rate constant, (iv) SOCS1 protein degradation rate, (v) STAT1 unbinding rate from active receptor-SOCS1 complexes, and (vi) set the EIF4E-dependent translation rate constant of SOCS1 to zero (see (40) for details on the EIF4E effect on translation). We fit the chosen parameters individually, while keeping the values of "best fit" at each step. The range of variation was set at \pm 2 in $\log 10$ scale. The 
851 SPARCED-I model was then run 1000 simulated hours, without any ligand stimulation, for

852 equilibration.

853 Context estimation of the SPARCED-I model

854 The SPARCED model initial conditions are based on serum and growth factor starved MCF10A 855 cells, grown in standard tissue culture plates. However, the experiments involving IFN $\gamma$ were done

856 in media with horse serum and collagen-coated tissue culture plates. The details of both 857 experimental procedures are explained below. "Bridge" experiments showed that the horse serum 858 upregulates active AKT (ppAKT) levels four-fold prior to growth factor stimulation. We modeled 859 increased basal levels of active AKT with IGF1 treatment for 24 hours, and found that $0.02 \mathrm{nM}$ 860 IGF1 was consistent with the above experimental observations (Figs. S16 and S17). Therefore, 861 we simulate the SPARCED-I model with 0.02 nM IGF1 treatment for 24 hours prior to EGF or 862 EGF+IFN $\gamma$ stimulation.

Cell proliferation estimation in SPARCED-I model

864 The Bouhaddou2018 model used cyclin A levels as a proxy for cell cycle entry in each cell (40). 865 Here, we used the same MATLAB scripts (findpeaks.m) as the Bouhaddou2018 model to 866 identify the peaks in cyclin A trajectories. A peak is defined as a hill-like shape, with a minimum 867 prominence of $4 \mathrm{nM}$ compared to surrounding basal levels. For each cell, the number of 868 occurrences of such peaks are counted within 0-48 hours of simulation. Then, the peak counts in 869 all 100 starting cells are summed to find the total number cell cycles in the population.

870 SPARCED-nf (nextflow, model version running on Nautilus-Kubernetes cluster)

871 The SPARCED model can be ported to high-performance cloud computing infrastructures for 872 large-scale simulation, in the SPARCED-nf variant. Specifically, we ran SPARCED-nf simulations 873 using the Pacific Research Platform, a distributed network of academic computing resources 874 organized as a Kubernetes cluster $(64,66)$. The prevalence of Kubernetes on both democratized 
875 and commercial cloud compute networks makes the model portable, allowing users to run large-

876 scale jobs on distributed supercomputers on a wide range of platforms (65).

877 Dependencies

878 (1) Docker: As is the practice with Kubernetes-compatible workflows, all model dependencies 879 and runtime environments are Dockerized into a downloadable image for self-contained model execution. This means when a job for SPARCED-nf is launched on the Kubernetes cluster, it will download the Docker image for SPARCED-nf and execute the model within that container. The

882 Docker image for SPARCED-nf is built on the Ubuntu-18.04 operating system with python3 https://hub.docker.com/repository/docker/birtwistlelab/sparced. converted into python source code and re-modularized for greater parallel-simulation efficiency. The process of creating and executing the model is handled entirely by Nextflow, a workflowmanagement application and language for building resilient pipelines. When SPARCED-nf is launched, Nextflow begins by creating a head pod on the cluster to coordinate each of the jobs needed to run the model (Fig. S1). The head pod creates smaller jobs that each download the containerized dependencies from Dockerhub, pull the model source files from the SPARCED-nf

892 GitHub repository, and run the assigned process. Once the model has completed execution, the 893 output files are saved to a section of the Kubernetes cluster called the persistent volume claim 894 (PVC), where they remain stored in the cloud for user download. 
automating common PVC tasks with Kubernetes' kubectl tool. The kube-load. sh file is used

900 to write new input to the PVC, and kube-login. sh is used to access and delete old input files

901 from the cluster.

902

Along with its scalability, SPARCED-nf is also highly customizable. The nextflow.config configuration file is used to define the specifics of simulation scenarios.

904 (1) nextflow. config: This configuration file has two main sections. In the first section (called $905 \mathrm{~K} 8)$, users define the Kubernetes namespace specifics and folder configurations. In the second 906 section (called params), users customize runtime arguments for simulation settings. The available parameters are input_dir_name (the directory name of the input files), flag_deterministic (flag=1 for deterministic or flag=0 for hybrid simulations), sim_time (simulation time in hours), Vol_nuclear (volume of nuclear compartment in liters), Vol_cyto (volume of cytoplasmic compartment in liters), speciesVals (species names + initial concentration values to start from), ratelawVals (parameter names + values), and numCells (number of single cells if the simulations are hybrid). Importantly, the "speciesVals" and "ratelawVals" parameters allow users to pass in a formatted string to specify parameter sweeps. Using these in conjunction with the "numCells" parameter, the user can

914 simulate thousands of cells in hundreds of different microenvironments in a single execution.

(2) SPARCED-nf:model_build: Analogous to "createModel.ipynb" in SPARCED-jupyter model, this phase of the Nextflow pipeline constructs all necessary files for the model simulation. (3) SPARCED-nf:split_from_params: This is the major parallelizing step of SPARCED-nf.

918 Having received the relevant model files from the last step, the workflow ingests the speciesVals,

919 ratelawVals, and numCells arguments set by the user in the nextflow. config. Using the input 920 files, it creates new input files to satisfy the user-specified parameter sweeps. Each new input file permutation is moved into its own new folder, and each such folder is duplicated numCells times. 
922 (4) SPARCED-nf:model_run: This final step of the Nextflow workflow is responsible for model 923 execution and output generation. Each folder created in the previous step above serves as the 924 unique runtime environment in this step. The model pulls assigned simulation input files 925 associated with the folder. Each instance of this step is run in parallel across different simulation 926 environments (Fig. S1B). Functionally, the code executed is very similar to the 927 "runModel. ipynb" notebook and the model outputs are saved to the PVC.

928 When the models complete execution, each SPARCED-nf:model_run instance saves its output

929 to a unique folder on the PVC. To download these folders to the local filesystem, users can employ 930 kube-save.sh (from the kube-runner module).

931 GitHub

932 The final model scripts, files, and information are available in Birtwistle Lab GitHub repository, 933 github.com/birtwistlelab/SPARCED.

934 Experimental Methods

935 Cell culture and western blotting

936 Cell culture: MCF10A cells (acquired from LINCS Consortium/Gordon Mills and STR verified 937 internally) are cultured in DMEM/F12 (Gibco \#11330032) medium supplemented with 5\% (by 938 volume) horse serum (Gibco \#16050122), 20ng/mL EGF (PeproTech \#AF-100-15), 0.5 mg/mL

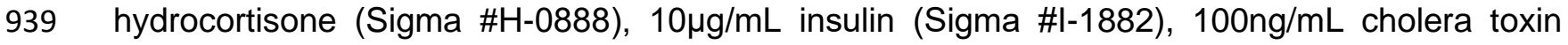
940 (Sigma \#C-8052), and 2mM L-Glutamine (Corning \#25-005-Cl). Cells were cultured at $37^{\circ} \mathrm{C}$ in $9415 \%$ CO2 in a humidified incubator and passaged every $2-3$ days with $0.25 \%$ trypsin (Corning \#25942 053-Cl) to maintain subconfluency. Serum starvation medium is DMEM/F12 medium 943 supplemented with $2 \mathrm{mM}$ L-Glutamine. Experimental starvation medium is DMEM/F12 medium 944 supplemented with 5\% (by volume) horse serum (Gibco \#16050122), $0.5 \mathrm{mg} / \mathrm{mL}$ hydrocortisone 
945 (Sigma \#H-0888), 100ng/mL cholera toxin (Sigma \#C-8052), and 2mM L-Glutamine (Corning \#25$946 \quad 005-\mathrm{Cl})$.

947 Tissue culture treated, non-collagen coated plates with full serum starvation: The cells were 948 seeded in full growth media at 150,000 cells/well in tissue culture treated six well plates (Corning \# 08-772-1B). The next day, cells are washed once with 1X PBS (one phosphate buffered saline tablet (Sigma \#P4417-100TAB) in $200 \mathrm{~mL}$ milli-Q water, autoclaved) and the media was exchanged to serum starvation media (DMEM/F12 medium, 2mM L-Glutamine) for 16-24 hours.

952 Then, the cells were treated with vehicle control, EGF (10 ng/mL, PeproTech \#AF-100-15), and HGF (40 ng/mL, R\&D Systems \#294-HGN-005) for 0, 5, and 60 minutes in a humidified, 5\% CO2, $37^{\circ} \mathrm{C}$ incubator.

Collagen-coated plates and growth-factor starvation only: Collagen-coating mixture was prepared as follows: $7.5 \mathrm{~mL}$ diluent buffer (20\% v/v glycerol, $10 \mathrm{mM}$ EDTA, PBS), $1.5 \mathrm{~mL}$ Tris-HCL, $0.6 \mathrm{~mL}$

957 COL1 (Cultrex \#3442-050-01), and 5.4 mL PBS. $950 \mu \mathrm{L}$ coating mix was added into each well of 958 a six-well plate. After making sure that the entire well surface was covered, the plates were 959 incubated one hour at room temperature. After incubation, any remaining liquid is aspirated and discarded. The wells were washed twice with sterile PBS and left lid-open under a sterile laminar

961 flow hood until wells were fully dry ( one hour). Upon replacement of the plate lid, the plates were stored in a benchtop desiccator at room temperature for a minimum of 3 days before use. Then, MCF10A cells were seeded in full growth media at 150,000 cells/well. After being allowed to attach for 7-8 hours, the wells were washed once with PBS and the media was changed to full growth media without EGF and insulin for 18 hours. Then, the cells were treated with vehicle control, EGF (10 ng/mL), and HGF (40 ng/mL) as above for 0,5 , and 60 minutes. ice. The media in the wells were aspirated and the wells were washed with PBS. $110 \mu \mathrm{L}$ of freshlyprepared, ice-cold RIPA buffer (50mM Tris, pH 7-8 (Acros Organics \#14050-0010), $150 \mathrm{mM} \mathrm{NaCl}$ 
970 (Fluka \#71383), $0.1 \%$ SDS (Fisher \#46040CI), 0.5\% sodium deoxycholate, 1\% Triton-X-100, filter

971 sterilized, stored at $4 \mathrm{C})$ with protease \& phosphatase inhibitors $(1 \mu \mathrm{g} / \mathrm{mL}$ aprotinin, $1 \mu \mathrm{g} / \mathrm{mL}$

972 leupeptin, $1 \mu \mathrm{g} / \mathrm{mL}$ pepstatin A, $10 \mathrm{mM} \beta$-glycerophosphate, and $1 \mathrm{mM}$ sodium orthovanadate) was

973 added into each well, while gently rotating the plate to cover the full surface area. The plates were

974 transferred to the cold room for 15-20 minutes, with slow rocking. The lysate was scraped off from

975 the wells with a cell scraper. $100 \mu \mathrm{L}$ of cell lysate from each well were transferred into labeled

976 Eppendorf tubes on ice. Each tube was vortexed three times to homogenize cell debris, keeping

977 other tubes on ice. All tubes were then centrifuged at $4^{\circ} \mathrm{C}$ for 15 minutes at $14000 \mathrm{rpm} .80 \mu \mathrm{L}$ of

978 the supernatant from each tube was transferred into new Eppendorf tubes on ice. These cleared

979 lysate samples were stored at -80C for long-term storage or used immediately as below.

Protein quantification: Total protein quantification was done using the BCA-Pierce 660 Assay (Thermo Scientific \#23225). As the reference, BSA stock (Thermo Scientific \#23209) was used according to the manufacturer protocol. In short, $10 \mu \mathrm{L}$ of samples and BSA standards were loaded into wells, in triplicate, in a 96-well plate (Corning \#3370). $150 \mu \mathrm{L}$ BCA Protein Assay Reagent was loaded into each non-empty well. The plate was covered and incubated at room temperature for 5 minutes. The absorbance at $660 \mathrm{~nm}$ was measured on a plate reader (BioTek \#Epoch2). The average reading of blank wells was subtracted from all other readings, and then average readings were calculated. The standard curve was fitted by a polynomial using blankcorrected mean values of each standard condition versus its BSA concentration. The fitted curve was used to determine the protein concentration in each sample.

Immunoblotting and quantification: The lysates were put on ice and the amount of sample to load into each well was calculated using the total protein concentrations determined above $(3 \mu \mathrm{q}$ loaded here). Each sample was mixed with $2 X$ Sample Buffer $(950 \mu \mathrm{L}$ of Laemmli's Buffer (BioRad \#161-0737), 50 LL beta-mercaptoethanol (Fisher \#03446I-100)) in a 1:1 ratio and transferred into a new Eppendorf tube. The sample solutions were heated at $95^{\circ} \mathrm{C}$ for $5 \mathrm{~min}$ on the heating plate 
and then briefly spun in benchtop microcentrifuge to return any condensation to the bottom of the tube. $10 \%$ acrylamide gels were prepared, and samples were loaded into the wells. A pre-stained protein ladder (LI-COR \#928-70000) was loaded in the first and last wells. The gel was run in SDS running buffer (100 mL 10X Tris-Glycine-SDS buffer (IBI Scientific \#IB01160) + $900 \mathrm{~mL}$ milli-Q water) at constant $220 \mathrm{~V}$ until the dye front runs off the gel. Then, wet-transfer to nitrocellulose membrane $(0.45 \mu \mathrm{m}$ pore size, VWR \#10063-173) was done using cold transfer buffer (3.03g Trisbase (Acros Organics \#14050-0010), 14.4g glycine (Acros Organics \#220910050), $100 \mathrm{~mL}$ methanol (BDH \#BDH1135-4LP), volume adjusted to 1 Liter with milli-Q water) and running the cassette with ice block at constant 100V for one hour. 1X TBST (Tris-Buffered Saline, 0.1\% Tween) was prepared: $100 \mathrm{~mL}$ of $10 X$ TBS solution (24 g Tris-base, $88 \mathrm{~g} \mathrm{NaCl}$ (Fluka \#71383), adjusted $\mathrm{pH}$ to 7.6 , adjusted final volume to 1 Liter with milli-Q water, autoclaved), $1 \mathrm{~mL}$ Tween20 (Fisher \#BP337-100), and milli-Q water until final volume of 1 Liter ( 900 mL). When the transfer was finished, the membrane was blocked using BSA-TBST blocking buffer ( $2.5 \mathrm{~g}$ bovine serum albumin (Fisher\# BP1600-100), $50 \mathrm{~mL} 1 \mathrm{X}$ TBST) for 45min at room temperature. The blocking buffer was discarded, and the membrane is incubated in primary antibody solution (1:1000 dilution, $10 \mu \mathrm{L}$ primary antibody in $10 \mathrm{~mL}$ 5\% BSA-TBST blocking buffer) overnight in cold room. The primary antibodies used were: AKT_pS473 (Cell Signaling \#4060; 1:1000), AKT (Cell Signaling \#2920; 1:1000), ERK_pT202_pY204 (Cell Signaling \#4370; 1:1000), ERK (Cell Signaling \#4696; 1:1000), alpha-tubulin (Novus \#NB100-690, 1:1000), and $\beta$-actin (LI-COR \#92642212, 1:1000). After primary antibody incubation, membranes were washed three times for 15 min each with $1 \mathrm{X}$ TBST at room temperature, with gentle rocking. Then, the membranes are incubated with LI-COR secondary antibodies in $10 \mathrm{ml}$ TBST blocking buffer for 45 minutes (antirabbit 800CW, LI-COR \#926-32211 or anti-mouse 680LT, LI-COR \#925-68070; $1: 8000$ ) at room temperature, with gentle rocking. Membranes was washed three times for 15 min each, with $1 \mathrm{X}$ TBST, on rocker. The imaging was done with a LI-COR Odyssey Infrared Imager, where bands were quantified using LI-COR Image Studio Lite v5.2 software (Figs. S16-19). 
bioRxiv preprint doi: https://doi.org/10.1101/2020.11.09.373407; this version posted July 15, 2021. The copyright holder for this preprint (which was not certified by peer review) is the author/funder. All rights reserved. No reuse allowed without permission.

\section{Data availability}

1022 The LINCS datasets analyzed during the current study are available in the Synapse repository,

1023 synapse.org/\#!Synapse:syn12526172. All other data are available from the authors upon 1024 reasonable request.

1025 


\section{ACKNOWLEDGMENTS}

1027 The authors acknowledge funding from the National Institutes of Health Grants R01GM104184 1028 and U54HG008098-LINCS Center (MRB), U54CA209988 and U54HG008100-LINCS Center $1029(\mathrm{LMH})$, the National Science Foundation Grant $\mathrm{CC}^{*}-1659300$ (FAF), and portions of this work 1030 were performed under the auspices of the U.S. Department of Energy by Lawrence Livermore 1031 National Laboratory under Contract DE-AC52-07NA27344 (RCB). CE was an NIH-LINCS 1032 Postdoctoral Fellow.

1033

1034 DECLARATION OF INTERESTS

1035 The authors declare no competing interests. 


\section{AUTHOR CONTRIBUTIONS}

1038 Conceptualization: CE, MRB

1039 Methodology: CE, LMH, FAF, MRB

1040 Software: CE, EMB, AM, MMS, MB, RCB, WD

1041 Validation: CE, EMB, AM, RCB, MRB

1042 Formal Analysis: CE

1043 Investigation: CE, SMG, LMH

1044 Resources: LMH, FAF, MRB

1045 Data Curation: CE, AM, WD, SMG

1046 Writing - Original Draft: CE, MRB

1047 Writing - Review \& Editing: CE, MRB

1048 Visualization: CE, EMB, MB

1049 Supervision: CE, MRB

1050 Project Administration: CE, MRB

1051 Funding Acquisition: LMH, FAF, MRB 
1053

1054

1055

1056

1057

1058

1059

1060

1061

1062

1063

1064

1065

1066

1067

1068

1069

1070

1071

1072

1073

1074

1075

1076

1077

1078

1079

1080

1081

1082

1083

1084

1085

1086

1087

1088

\section{REFERENCES}

1. Wilkinson MD, Dumontier M, Aalbersberg ljJ, Appleton G, Axton M, Baak A, et al. The FAIR Guiding Principles for scientific data management and stewardship. Sci Data. 2016 Dec;3(1):160018.

2. Ma'ayan A, Rouillard AD, Clark NR, Wang Z, Duan Q, Kou Y. Lean Big Data integration in systems biology and systems pharmacology. Trends Pharmacol Sci. 2014 Sep;35(9):450-60.

3. Gomez-Cabrero D, Abugessaisa I, Maier D, Teschendorff A, Merkenschlager M, Gisel A, et al. Data integration in the era of omics: current and future challenges. BMC Syst Biol. 2014;8(Suppl 2):I1.

4. Stites EC, Aziz M, Creamer MS, Von Hoff DD, Posner RG, Hlavacek WS. Use of Mechanistic Models to Integrate and Analyze Multiple Proteomic Datasets. Biophys J. 2015 Apr;108(7):1819-29.

5. Mirza B, Wang W, Wang J, Choi H, Chung NC, Ping P. Machine Learning and Integrative Analysis of Biomedical Big Data. Genes. 2019 Jan 28;10(2):87.

6. Huang S, Chaudhary K, Garmire LX. More Is Better: Recent Progress in Multi-Omics Data Integration Methods. Front Genet. 2017 Jun 16;8:84.

7. Zeng ISL, Lumley T. Review of Statistical Learning Methods in Integrated Omics Studies (An Integrated Information Science). Bioinforma Biol Insights. 2018 Jan;12:117793221875929.

8. Jensen $\mathrm{KJ}$, Janes KA. Modeling the latent dimensions of multivariate signaling datasets. Phys Biol. 2012 Aug 1;9(4):045004.

9. Adam G, Rampášek L, Safikhani Z, Smirnov P, Haibe-Kains B, Goldenberg A. Machine learning approaches to drug response prediction: challenges and recent progress. Npj Precis Oncol. 2020 Dec;4(1):19.

10. lanevski A, Giri AK, Gautam P, Kononov A, Potdar S, Saarela J, et al. Prediction of drug combination effects with a minimal set of experiments. Nat Mach Intell. 2019 Dec;1(12):568-77.

11. Liu H, Zhang W, Nie L, Ding X, Luo J, Zou L. Predicting effective drug combinations using gradient tree boosting based on features extracted from drug-protein heterogeneous network. BMC Bioinformatics. 2019 Dec;20(1):645.

12. Wong D, Yip S. Machine learning classifies cancer. Nature. 2018 Mar;555(7697):446-7.

13. Ehteshami Bejnordi B, Veta $M$, Johannes van Diest $P$, van Ginneken B, Karssemeijer N, Litjens G, et al. Diagnostic Assessment of Deep Learning Algorithms for Detection of Lymph Node Metastases in Women With Breast Cancer. JAMA. 2017 Dec 12;318(22):2199. 
14. Kleppe A, Albregtsen F, Vlatkovic L, Pradhan M, Nielsen B, Hveem TS, et al. Chromatin organisation and cancer prognosis: a pan-cancer study. Lancet Oncol. 2018 Mar;19(3):356-69.

15. Esteva A, Kuprel B, Novoa RA, Ko J, Swetter SM, Blau HM, et al. Dermatologist-level classification of skin cancer with deep neural networks. Nature. 2017 Feb 2;542(7639):115-8.

16. Yu MK, Ma J, Fisher J, Kreisberg JF, Raphael BJ, Ideker T. Visible Machine Learning for Biomedicine. Cell. 2018 Jun;173(7):1562-5.

17. Baker RE, Peña J-M, Jayamohan J, Jérusalem A. Mechanistic models versus machine learning, a fight worth fighting for the biological community? Biol Lett. 2018 May;14(5):20170660.

18. Najafabadi MM, Villanustre F, Khoshgoftaar TM, Seliya N, Wald R, Muharemagic E. Deep learning applications and challenges in big data analytics. J Big Data. 2015 Dec;2(1):1.

19. Wang F, Casalino LP, Khullar D. Deep Learning in Medicine-Promise, Progress, and Challenges. JAMA Intern Med. 2019 Mar 1;179(3):293.

20. Yang JH, Wright SN, Hamblin M, McCloskey D, Alcantar MA, Schrübbers L, et al. A White-Box Machine Learning Approach for Revealing Antibiotic Mechanisms of Action. Cell. 2019 May;177(6):1649-1661.e9.

21. Kholodenko BN, Hancock JF, Kolch W. Signalling ballet in space and time. Nat Rev Mol Cell Biol. 2010;11:414-26.

22. Carrera J, Covert MW. Why Build Whole-Cell Models? Trends Cell Biol. 2015 Dec;25(12):719-22.

23. Karr JR, Sanghvi JC, Macklin DN, Gutschow MV, Jacobs JM, Bolival Jr. B, et al. A wholecell computational model predicts phenotype from genotype. Cell. 2012;150:389-401.

24. Carrera J, Elena SF, Jaramillo A. Computational design of genomic transcriptional networks with adaptation to varying environments. Proc Natl Acad Sci. 2012 Sep 18;109(38):15277-82.

25. Münzner U, Klipp E, Krantz M. A comprehensive, mechanistically detailed, and executable model of the cell division cycle in Saccharomyces cerevisiae. Nat Commun. 2019 Dec;10(1):1308.

26. Saez-Rodriguez J, Blüthgen N. Personalized signaling models for personalized treatments. Mol Syst Biol [Internet]. 2020 Jan [cited 2020 Jul 27];16(1). Available from: https://onlinelibrary.wiley.com/doi/abs/10.15252/msb.20199042

27. Halasz M, Kholodenko BN, Kolch W, Santra T. Integrating network reconstruction with mechanistic modeling to predict cancer therapies. Sci Signal. 2016;9(455):ra114. 
28. Macklin DN, Ahn-Horst TA, Choi H, Ruggero NA, Carrera J, Mason JC, et al. Simultaneous cross-evaluation of heterogeneous E. coli datasets via mechanistic simulation. Science. 2020 24;369(6502).

29. Santos SDM, Verveer PJ, Bastiaens PIH. Growth factor-induced MAPK network topology shapes Erk response determining PC-12 cell fate. Nat Cell Biol. 2007 Mar;9(3):324-30.

30. Kholodenko BN, Demin OV, Moehren G, Hoek JB. Quantification of short term signaling by the epidermal growth factor receptor. J Biol Chem. 1999 Oct 15;274(42):30169-81.

31. Tyson JJ. Modeling the cell division cycle: cdc2 and cyclin interactions. Proc Natl Acad Sci. 1991 Aug 15;88(16):7328-32.

32. Nyman E, Fagerholm S, Jullesson D, Stralfors P, Cedersund G. Mechanistic explanations for counter-intuitive phosphorylation dynamics of the insulin receptor and insulin receptor substrate-1 in response to insulin in murine adipocytes. Febs J. 2012;279:987-99.

33. Schmierer B, Tournier AL, Bates PA, Hill CS. Mathematical modeling identifies Smad nucleocytoplasmic shuttling as a dynamic signal-interpreting system. Proc Natl Acad Sci. 2008 May 6;105(18):6608-13.

34. Vilar JMG, Guet CC, Leibler S. Modeling network dynamics. J Cell Biol. 2003 May 12;161(3):471-6.

35. Kofahl B, Klipp E. Modelling the dynamics of the yeast pheromone pathway. Yeast. 2004 Jul 30;21(10):831-50.

36. Tyson JJ, Chen K, Novak B. Network dynamics and cell physiology. Nat Rev Mol Cell Biol. 2001 Dec;2(12):908-16.

37. Puszyński K, Hat B, Lipniacki T. Oscillations and bistability in the stochastic model of p53 regulation. J Theor Biol. 2008 Sep;254(2):452-65.

38. Sedaghat AR, Sherman A, Quon MJ. A mathematical model of metabolic insulin signaling pathways. Am J Physiol Endocrinol Metab. 2002;283:E1084-101.

39. Carrera J, Estrela R, Luo J, Rai N, Tsoukalas A, Tagkopoulos I. An integrative, multiscale, genome-wide model reveals the phenotypic landscape of $E$ scherichia coli . Mol Syst Biol. 2014 Jul;10(7):735.

40. Bouhaddou M, Barrette AM, Stern AD, Koch RJ, DiStefano MS, Riesel EA, et al. A mechanistic pan-cancer pathway model informed by multi-omics data interprets stochastic cell fate responses to drugs and mitogens. PLoS Comput Biol [Internet]. 2018;14(3). Available from:

http://journals.plos.org/ploscompbiol/article/file?id=10.1371/journal.pcbi.1005985\&type=pr intable

41. Fröhlich F, Kessler T, Weindl D, Shadrin A, Schmiester L, Hache H, et al. Efficient Parameter Estimation Enables the Prediction of Drug Response Using a Mechanistic Pan-Cancer Pathway Model. Cell Syst. 2018 Dec;7(6):567-579.e6. 
42. Dalle Pezze P, Sonntag AG, Thien A, Prentzell MT, Godel M, Fischer S, et al. A Dynamic Network Model of mTOR Signaling Reveals TSC-Independent mTORC2 Regulation. Sci Signal. 2012 Mar 27;5(217):ra25-ra25.

43. Capuani F, Conte A, Argenzio E, Marchetti L, Priami C, Polo S, et al. Quantitative analysis reveals how EGFR activation and downregulation are coupled in normal but not in cancer cells. Nat Commun. 2015 Nov;6(1):7999.

44. Orth JD, Thiele I, Palsson BØ. What is flux balance analysis? Nat Biotechnol. 2010 Mar;28(3):245-8.

45. Lee JM. Flux balance analysis in the era of metabolomics. Brief Bioinform. 2006 Mar 7;7(2):140-50.

46. Sherman MS, Cohen BA. A Computational Framework for Analyzing Stochasticity in

47. Raj A, Peskin CS, Tranchina D, Vargas DY, Tyagi S. Stochastic mRNA Synthesis in Mammalian Cells. Schibler U, editor. PLoS Biol. 2006 Sep 12;4(10):e309.

48. Raj A, van Oudenaarden A. Nature, Nurture, or Chance: Stochastic Gene Expression and Its Consequences. Cell. 2008 Oct;135(2):216-26.

49. Faeder JR, Blinov ML, Goldstein B, Hlavacek WS. Rule-based modeling of biochemical networks. Complexity. 2005 Mar;10(4):22-41.

50. Harris LA, Hogg JS, Tapia JJ, Sekar JA, Gupta S, Korsunsky I, et al. BioNetGen 2.2: advances in rule-based modeling. Bioinformatics. 2016;32:3366-8.

51. Xu W, Smith AM, Faeder JR, Marai GE. RuleBender: a visual interface for rule-based modeling. Bioinformatics. 2011;27:1721-2.

52. Boutillier P, Maasha M, Li X, Medina-Abarca HF, Krivine J, Feret J, et al. The Kappa platform for rule-based modeling. Bioinformatics. 2018 Jul 1;34(13):i583-92.

53. Lopez CF, Muhlich JL, Bachman JA, Sorger PK. Programming biological models in Python using PySB. Mol Syst Biol. 2013;9.

54. Sneddon MW, Faeder JR, Emonet T. Efficient modeling, simulation and coarse-graining of biological complexity with NFsim. Nat Methods. 2011 Feb;8(2):177-83.

55. Hogg JS, Harris LA, Stover LJ, Nair NS, Faeder JR. Exact hybrid particle/population simulation of rule-based models of biochemical systems. PLoS Comput Biol. 2014 Apr;10(4):e1003544. 20. 
58. Azeloglu EU, lyengar R. Good practices for building dynamical models in systems biology. Sci Signal. 2015;8.

59. Hucka M, Finney A, Sauro HM, Bolouri H, Doyle JC, Kitano H, et al. The systems biology markup language (SBML): a medium for representation and exchange of biochemical network models. Bioinformatics. 2003 Mar 1;19(4):524-31.

1201

1202

1203

1204

1205

1206

1207

1208

1209

1210

1211

1212

64. Rensin DK. Kubernetes-scheduling the future at cloud scale. 2015;

1213

1214

1215

1216

1217

1218

1219

1220

1221

1222

1223

1224

1225

1226

1227

1228

1229

1230

1231

1232

65. Thurgood B, Lennon RG. Cloud Computing With Kubernetes Cluster Elastic Scaling. In: Proceedings of the 3rd International Conference on Future Networks and Distributed Systems - ICFNDS '19 [Internet]. Paris, France: ACM Press; 2019 [cited 2020 Sep 22]. p. 1-7. Available from: http://dl.acm.org/citation.cfm?doid=3341325.3341995

66. Smarr L, Crittenden C, DeFanti T, Graham J, Mishin D, Moore R, et al. The Pacific Research Platform: Making High-Speed Networking a Reality for the Scientist. In: Proceedings of the Practice and Experience on Advanced Research Computing [Internet]. Pittsburgh PA USA: ACM; 2018 [cited 2020 Sep 22]. p. 1-8. Available from: https://dl.acm.org/doi/10.1145/3219104.3219108

67. Fröhlich F, Theis FJ, Rädler JO, Hasenauer J. Parameter estimation for dynamical systems with discrete events and logical operations. Bioinformatics. 2016 Dec 31;btw764.

68. Kluyver T, Ragan-Kelley B, Pérez F, Granger B, Bussonnier M, Frederic J, et al. Jupyter Notebooks - a publishing format for reproducible computational workflows. In: Loizides F, Schmidt B, editors. Positioning and Power in Academic Publishing: Players, Agents and Agendas. IOS Press; 2016. p. 87-90.

69. Fröhlich F, Kaltenbacher B, Theis FJ, Hasenauer J. Scalable Parameter Estimation for Genome-Scale Biochemical Reaction Networks. Stelling J, editor. PLOS Comput Biol. 2017 Jan 23;13(1):e1005331.

70. Nakakuki T, Birtwistle MR, Saeki Y, Yumoto N, Ide K, Nagashima T, et al. Ligand-Specific c-Fos Expression Emerges from the Spatiotemporal Control of ErbB Network Dynamics. Cell. 2010 May;141(5):884-96. 
71. von Kriegsheim A, Baiocchi D, Birtwistle M, Sumpton D, Bienvenut W, Morrice N, et al. Cell fate decisions are specified by the dynamic ERK interactome. Nat Cell Biol. 2009 Dec;11(12):1458-64.

72. Sullivan DM, Latham MD, Ross WE. Proliferation-dependent topoisomerase II content as a determinant of antineoplastic drug action in human, mouse, and Chinese hamster ovary cells. Cancer Res. 1987 Aug 1;47(15):3973-9.

73. Yamada S, Shiono S, Joo A, Yoshimura A. Control mechanism of JAK/STAT signal transduction pathway. FEBS Lett. 2003 Jan 16;534(1-3):190-6.

74. Torgerson TR, Colosia AD, Donahue JP, Lin YZ, Hawiger J. Regulation of NF-kappa B, AP-1, NFAT, and STAT1 nuclear import in T lymphocytes by noninvasive delivery of peptide carrying the nuclear localization sequence of NF-kappa B p50. J Immunol Baltim Md 1950. 1998 Dec 1;161(11):6084-92.

75. Tsai C-C, Kai J-I, Huang W-C, Wang C-Y, Wang Y, Chen C-L, et al. Glycogen Synthase Kinase-3 $\beta$ Facilitates IFN- $\gamma$-Induced STAT1 Activation by Regulating Src Homology-2 Domain-Containing Phosphatase 2. J Immunol. 2009 Jul 15;183(2):856-64.

76. Angel $\mathrm{P}$, Hattori $\mathrm{K}, \mathrm{Smeal} \mathrm{T}$, Karin $\mathrm{M}$. The jun proto-oncogene is positively autoregulated by its product, Jun/AP-1. Cell. 1988 Dec 2;55(5):875-85.

77. MeissI K, Macho-Maschler S, Müller M, Strobl B. The good and the bad faces of STAT1 in solid tumours. Cytokine. 2017 Jan;89:12-20.

78. Schroder K, Hertzog PJ, Ravasi T, Hume DA. Interferon- $\gamma$ : an overview of signals, mechanisms and functions. J Leukoc Biol. 2004 Feb;75(2):163-89.

79. Parker BS, Rautela J, Hertzog PJ. Antitumour actions of interferons: implications for cancer therapy. Nat Rev Cancer. 2016 Mar;16(3):131-44.

80. Townsend PA, Scarabelli TM, Davidson SM, Knight RA, Latchman DS, Stephanou A. STAT-1 Interacts with p53 to Enhance DNA Damage-induced Apoptosis. J Biol Chem. 2004 Feb 13;279(7):5811-20.

81. Koromilas AE, SexI V. The tumor suppressor function of STAT1 in breast cancer. JAKSTAT. 2013 Apr;2(2):e23353.

82. Seif F, Khoshmirsafa M, Aazami H, Mohsenzadegan M, Sedighi G, Bahar M. The role of JAK-STAT signaling pathway and its regulators in the fate of Thelper cells. Cell Commun Signal. 2017 Dec;15(1):23.

83. Hilton DJ. Negative regulators of cytokine signal transduction. Cell Mol Life Sci CMLS. 1999 Oct 2;55(12):1568-77.

84. Pawson T, Gish GD, Nash P. SH2 domains, interaction modules and cellular wiring. Trends Cell Biol. 2001 Dec 1;11(12):504-11.

85. Huang H, Li L, Wu C, Schibli D, Colwill K, Ma S, et al. Defining the Specificity Space of the Human Src Homology 2 Domain. Mol Cell Proteomics. 2008 Apr;7(4):768-84. 
86. Böhmer F-D, Friedrich K. Protein tyrosine phosphatases as wardens of STAT signaling. JAK-STAT. 2014 Jan 21;3(1):e28087.

87. Tseng P-C, Huang W-C, Chen C-L, Sheu B-S, Shan Y-S, Tsai C-C, et al. Regulation of SHP2 by PTEN/AKT/GSK-3 $\beta$ signaling facilitates IFN- $\gamma$ resistance in hyperproliferating gastric cancer. Immunobiology. 2012 Sep;217(9):926-34.

88. Wang S, Wang N, Yu B, Cao M, Wang Y, Guo Y, et al. Circulating IGF-1 promotes prostate adenocarcinoma via FOXO3A/BIM signaling in a double-transgenic mouse model. Oncogene. 2019 Sep;38(36):6338-53.

89. Weeks KL, Bernardo BC, Ooi JYY, Patterson NL, McMullen JR. The IGF1-PI3K-Akt Signaling Pathway in Mediating Exercise-Induced Cardiac Hypertrophy and Protection. In: Xiao J, editor. Exercise for Cardiovascular Disease Prevention and Treatment: From Molecular to Clinical, Part 2 [Internet]. Singapore: Springer Singapore; 2017. p. 187-210. Available from: https://doi.org/10.1007/978-981-10-4304-8_12

90. Melnik BC, John SM, Schmitz G. Over-stimulation of insulin/IGF-1 signaling by Western diet may promote diseases of civilization: lessons learnt from Laron syndrome. Nutr Metab. 2011 Jun 24;8(1):41.

91. Fröhlich F, Weindl D, Schälte Y, Pathirana D, Paszkowski $Ł$, Lines GT, et al. AMICl: HighPerformance Sensitivity Analysis for Large Ordinary Differential Equation Models. ArXiv201209122 Q-Bio [Internet]. 2020 Dec 16 [cited 2021 Mar 25]; Available from: http://arxiv.org/abs/2012.09122

92. Barrette AM, Bouhaddou M, Birtwistle MR. Integrating Transcriptomic Data with Mechanistic Systems Pharmacology Models for Virtual Drug Combination Trials. ACS Chem Neurosci. 2018 Jan 17;9(1):118-29.

93. Barretina J, Caponigro G, Stransky N, Venkatesan K, Margolin AA, Kim S, et al. The Cancer Cell Line Encyclopedia enables predictive modelling of anticancer drug sensitivity. Nature. 2012 Mar;483(7391):603-7.

94. Cancer Genome Atlas Research N. Comprehensive genomic characterization defines human glioblastoma genes and core pathways. Nature. 2008;455:1061-8.

95. Cancer Genome Atlas Research N, Weinstein JN, Collisson EA, Mills GB, Shaw KR, Ozenberger BA, et al. The Cancer Genome Atlas Pan-Cancer analysis project. Nat Genet. 2013;45:1113-20.

96. Hutter C, Zenklusen JC. The Cancer Genome Atlas: Creating Lasting Value beyond Its Data. Cell. 2018 Apr;173(2):283-5.

97. Uhlen M, Fagerberg L, Hallstrom BM, Lindskog C, Oksvold P, Mardinoglu A, et al. Tissuebased map of the human proteome. Science. 2015 Jan 23;347(6220):1260419-1260419.

98. Thul PJ, Åkesson L, Wiking M, Mahdessian D, Geladaki A, Ait Blal H, et al. A subcellular map of the human proteome. Science. 2017 May 26;356(6340):eaal3321. 
99. Uhlen M, Zhang C, Lee S, Sjöstedt E, Fagerberg L, Bidkhori G, et al. A pathology atlas of the human cancer transcriptome. Science. 2017 Aug 18;357(6352):eaan2507.

100. Bairoch A. The Cellosaurus, a Cell-Line Knowledge Resource. J Biomol Tech JBT. 2018 Jul;29(2):25-38.

101. Gibson MA, Bruck J. Efficient Exact Stochastic Simulation of Chemical Systems with Many Species and Many Channels. J Phys Chem A. 2000 Mar;104(9):1876-89.

102. Ghaffarizadeh A, Heiland R, Friedman SH, Mumenthaler SM, Macklin P. PhysiCell: An open source physics-based cell simulator for 3-D multicellular systems. Poisot T, editor. PLOS Comput Biol. 2018 Feb 23;14(2):e1005991.

103. Swat MH, Thomas GL, Belmonte JM, Shirinifard A, Hmeljak D, Glazier JA. Multi-Scale Modeling of Tissues Using CompuCell3D. In: Methods in Cell Biology [Internet]. Elsevier; 2012 [cited 2021 Mar 26]. p. 325-66. Available from: https://linkinghub.elsevier.com/retrieve/pii/B9780123884039000138

104. Szigeti B, Roth YD, Sekar JAP, Goldberg AP, Pochiraju SC, Karr JR. A blueprint for human whole-cell modeling. Curr Opin Syst Biol. 2018 Feb;7:8-15.

105. Hindmarsh AC, Brown PN, Grant KE, Lee SL, Serban R, Shumaker DE, et al. SUNDIALS: Suite of nonlinear and differential/algebraic equation solvers. ACM Trans Math Softw TOMS. 2005 Sep;31(3):363-96.

106. Yates AD, Achuthan P, Akanni W, Allen J, Allen J, Alvarez-Jarreta J, et al. Ensembl 2020. Nucleic Acids Res. 2019 Nov 6;gkz966.

107. Braschi B, Denny P, Gray K, Jones T, Seal R, Tweedie S, et al. Genenames.org: the

108. Weindl D, Fröhlich F, Stapor P, Schälte Y, AMICI-Developer, LeonardSchmiester, et al. ICB-DCM/AMICI: AMICI v0.11.2 [Internet]. Zenodo; 2020 [cited 2020 Jul 27]. Available from: https://zenodo.org/record/3949231 


\section{SUPPLEMENTAL INFORMATION}

1336

Supplemental Figure 1. (A) SPARCED-nf implementation overview. (B) Details of SPARCED-nf

1337 pipeline.

1338

1339

1340

1341

1342

1343

1344

1345

1346

1347

1348

1349

1350

1351

1352

1353

1354

1355

1356

1357

1358

Supplemental Figure 2. SPARCED model includes a stochastic gene expression module. Two isoforms of ERK gene (MAPK1 and MAPK3) are activated randomly (A) and leads to two distinct mRNAs (B). The ERK1 and ERK2 mRNAs are translated into a single ERK protein (C). The trajectories are obtained from a stochastic single cell simulation with no growth factor stimulation for 24 hours.

Supplemental Figure 3. (A) Ligand-receptor binding and Hill coefficients for each pair in MCF10A context. The simulations capture literature knowledge. (B) The dynamics of activated EGFR (membrane-bound and internalized) dimers are recaptured by the SPARCED model, compared to Bouhaddou2018 model.

Supplemental Figure 4. Signaling dynamics of ppERK, ppAKT, and pEIF4EBP1 induced by EGF, Insulin, or EGF+Insulin treatment for 6 hours. Serum-starved MCF10A cells are stimulated with EGF $(0.01,0.1,1$, and $10 \mathrm{nM})$, Insulin $(0.17,1.7,17$, and $1721 \mathrm{nM})$, or EGF+Insulin $(0.01+0.17,10+0.17,0.01+1721$, and $10+1721 \mathrm{nM})$.

Supplemental Figure 5. Signaling dynamics of ppERK and ppAKT induced by EGF, Heregulin (NRG1), HGF, PDGF, FGF, IGF, and Insulin treatment for 2 hours. Serum-starved MCF10A cells are stimulated with corresponding ligands at a dose range of 0.001 to $1000 \mathrm{nM}$.

Supplemental Figure 6. (A) p53 is activated in response to double (middle) or/and single (top/bottom) stranded DNA break damage. When DNA break repair mechanism is turned on (orange curves), p53 activity (or oscillatory behavior) dies down. (B) Single cells show different levels of p53 response to DNA damage. Increasing DNA damage amount (top to bottom) leads to higher number of activated p53 peaks. (C) the number of p53 pulses increases with increasing 
DNA damage, whereas pulse height and width remain relatively constant (results based on simulations shown in B). Plots show mean \pm s.e.m.

1361 Supplemental Figure 7. (A) Increasing TRAIL dose decreases the time it takes to die (ttd) for 1362 the average cell. Representative cells trajectories are shown, where the cells are simulated deterministically with different doses of TRAIL until they die (or up to 100 hours). The time of death is defined by the amount of cleaved PARP (cPARP, y-axis) surpassing the amount uncleaved PARP. (B) Summary of ttd values for different TRAIL doses. (C) The fraction of surviving cells decreases as stimulated TRAIL dose increases. The red circles represent percentage of 1367 living cells when 20 stochastic single cells are simulated with specified TRAIL dosage for 5 hours. 1368 The black stars are experimental data from Bouhaddou 2018 model (D) Increasing ERK and AKT 1369 activity levels prolongs TRAIL induced time to death (blue curve), whereas increasing PUMA and 1370 NOXA expression levels decreases the time it takes for cells to die (red curve). Cells with specified 1371 alterations are compared to the cell stimulated with a low dose of TRAIL (black curve). cPARP 1372 levels are the proxy for cell death, where the cells go apoptosis when [cPARP]>[PARP].

1373 Supplemental Figure 8. (A) Increasing Cyclin D mRNA levels induces proper cyclin-CDK 1374 complex progression and oscillations for cell cycle entry. Plots show Cyclin D, E, A, and B 1375 concentrations when basal (blue), 10X basal (dark orange), and 60X basal (light orange) levels of Cyclin D mRNA (CYCD) are simulated. (B) The number of ribosomes in the cell doubles around 137720 hours. The cell is simulated with full growth condition (EGF=100 nM, NRG1=100 nM, HGF=100 nM, PDGF=100 nM, FGF=100 nM, IGF=100 nM, INS=100 nM).

1379 Supplemental Figure 9. Inhibition of AKT and ERK pathways together synergistically increase 1380 cell death, in EGF and insulin stimulated cells. Serum-starved MCF10A cells are simulated with 1381 following conditions: (A) No stimulation, (B) EGF=20ng/mL + Insulin=10 $\mu \mathrm{g} / \mathrm{mL}$, (C) EGF=20ng/mL

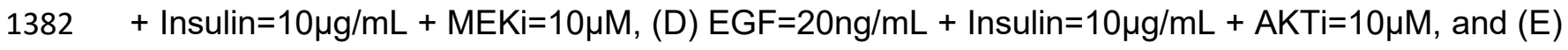


$E G F=20 n g / m L+$ Insulin=10 $\mu \mathrm{g} / \mathrm{mL}+\mathrm{MEKi}=10 \mu \mathrm{M}+\mathrm{AKTi}=10 \mu \mathrm{M}$ for up to 80 hours. The bar plots show mean \pm s.e.m. of time to death for 30 cells. The ttd are captured by cPARP spikes.

1385 Supplemental Figure 10. (A) Simulations where BIM-dependent or BAD-dependent mechanisms are switched off and percent death calculated in response to EGF + insulin at 48 hours. The results show that ERK and AKT inhibition induced cell death mechanisms are mostly BIM dependent, not BAD. Bars represent mean \pm s.e.m. of 100 stochastic cell simulations. (B) EGF and insulin cooperatively induce cell cycle entry, with insulin inducing very little cell cycle entry alone. Cells are simulated with EGF (10nM), Insulin (1721nM), or EGF+Insulin $(10 \mathrm{nM}+1721 \mathrm{nM})$ for 30 hours and the percentage of cells entering S-phase are calculated. Cells are considered in S-phase when the sum of concentrations of Cyclin $E, A$, and $B$ is greater than 20nM. Bars represent mean \pm s.e.m. of 100 stochastic cell simulations.

Supplemental Figure 11. Activation of both ERK and AKT pathways are required for robust cell cycle entry. Time averaged ppERK and ppAKT levels correlate with Cyclin D levels. Basal levels of ppERK and ppAKT are increased (between 1X-20X) and each condition is simulated up to 6

1397 hours. The time-averaged levels of ppERK and ppAKT are plotted against the time-averaged 1398 Cyclin D levels. Conditions representing EGF (10nM), Insulin (1721nM), and EGF+Insulin $1399(10 \mathrm{nM}+1721 \mathrm{nM})$ are shown with colored circles.

Supplemental Figure 12. SPARCED model recapitulates downstream pathway activation by 1401 ligands and ligand combination treatments. Experimental data and simulation results from 1402 MATLAB (lines) and SPARCED (circles) models with EGF (top) and EGF+Insulin (bottom) 1403 stimulation for 6 hours. Plots show double-phosphorylated ERK (ppERK), serine-phosphorylated 1404 AKT (pAKT), and phospho-EIF4EBP1 (pEIF4EBP1) levels. The numbers in gray shaded boxes 1405 represents numbering of conditions in Fig. 3A. Exp: Experimental data, Sim: Simulation. 
Supplemental Figure 13. (A) Bar plots corresponding to the conditions shown in Fig. 2C. Gray

1407 bars are experimental or simulation data from Bouhaddou 2018 model and blue bars are

1408 simulation results of SPARCED model. Bars represent mean \pm s.e.m. (B) Etoposide treatment

1409 alone induces lesser cell death compared to Etoposide + Growth Factor stimulation, shown in Fig.

1410 3D-E. (C) Percentage of cell death of 100 cells shown in (B). Bars represent mean \pm s.e.m.

1411 Supplemental Figure 14. SPARCED model alteration guidelines. (A) Steps of model 1412 expansion and context change procedures are listed. Refer to Supp. File 13 for more details. 1413 Steps can be skipped if no changes are necessary. (B) The list of parameters and species values 1414 modified for SPARCED model context change from MCF10A cells to U87 cells. (C) 1415 SPARCED_U87 model simulations reproduce previous observations, where U87 cells show 1416 increased response and sensitivity to AKT inhibition. MEKi: MEK inhibitor, AKTi: AKT inhibitor. 1417 Bars represent mean \pm s.e.m. of 100 single cell simulations for each condition.

1418 Supplemental Figure 15. Comparison of initialized species concentrations for MCF10A (top) and 1419 U87 (bottom) cells. The Bouhaddou2018 model values are reproduced by the new initialization 1420 notebook. The concentrations values (black dots) are almost exact and identity line (dashed red) 1421 coincides with the linear fit line (solid black). SPARCED initialized values are on y-axis and B2018 1422 initialized values are on $x$-axis.

1423 Supplemental Figure 16. (A) The experimental setup and (B) results of exploring the effect of 1424 collagen-coating and serum starvation in MCF10A cells.

1425 Supplemental Figure 17. (A) The quantification of western blots shown in Fig. S16. All data are 1426 normalized to condition 10 (cells in non-coated plates with full growth media, measurements at 1427 time $0 \mathrm{hr}$ ). At least three biological replicates. pAKT levels in growth factor starved cells in 1428 collagen-coated plates (\#3) are four times higher than pAKT levels in serum+growth factor starved 1429 cells in non-coated plates (\#11). No significant change in pERK levels between two conditions 
1430 (\#3 and \#11). The order of bars is given according to the list on the left in panel (B). (B) The 1431 experimental conditions of western blot membranes in Figs. S16, S18, and S19.

1432 Supplemental Figure 18. The full images of western blots (replicates 1 and 2) shown in Fig. S16.

1433 Supplemental Figure 19. The full images of western blots (replicates 3 and 4) shown in Fig. S16.

1434 Supplemental Figure 20. (A) p21 trajectories of SPARCED-I Mechanism 1 model simulations. 1435 Changing STAT1 regulation of p21 transcription induces higher p21 levels (orange, right) 1436 compared to original (orange, left) simulations. The p21 level increase does not occur in single 1437 EGF stimulation conditions (blue). (B) The total number of cyclin A peaks of 100 starting cells at 143848 hours in SPARCED-I mech1 (gray) and modified SPARCED-I mech1 (orange) models.

Supplemental Figure 21. (A-B) Normalized pMAPK levels show a significant change when IFN $\gamma$ 1440 is included in addition to the EGF, in both Mechanism 1 (A) and Mechanism 2 (B). (C) Normalized 1441 ppAKT levels do not show a significant decrease after IFN $\gamma$ treatment, when EGF+IFN $\gamma$ 1442 Mechanism 1 simulations are compared to EGF alone case. (D) Normalized ppAKT levels show 1443 a significant decrease after IFN $\gamma$ treatment, when EGF+IFN $\gamma$ Mechanism 2 simulations are 1444 compared to EGF alone case. RPPA data are shown in black error lines, from three independent 1445 replicates. Colored dark lines represent median cell trajectories from simulations, dark and light1446 colored regions represent 70th and 95th quantiles, respectively. Experimental data are from 1447 synapse.org/\#!Synapse:syn12526172.

1448 Supplemental File 1. OmicsData input file.

1449 Supplemental File 2. Species input file.

1450 Supplemental File 3. Ratelaws input file.

1451 Supplemental File 4. Stoichiometric Matrix input file.

1452 Supplemental File 5. Gene Regulation input file. 
1453 Supplemental File 6. Compartments input file.

1454 Supplemental File 7. Observables input file.

1455 Supplemental File 8. Model creation Jupyter notebook.

1456 Supplemental File 9. SPARCED model Antimony file.

1457 Supplemental File 10. SPARCED model SBML file.

1458 Supplemental File 11. Model import and simulation Jupyter notebook.

1459 Supplemental File 12. Parameter value replacements for U87 cell line SPARCED model.

1460 Supplemental File 13. SPARCED model alteration steps.

1461 Supplemental File 14. U87 cell line omics data and model parameter values from the 1462 Bouhaddou2018 model.

1463 Supplemental File 15. Protein-to-mRNA ratios file.

1464 Supplemental File 16. Model initialization Jupyter notebook.

1465 Supplemental File 17. Model initialization Jupyter notebook for U87 cell line.

1466 Supplemental File 18. Initialization input file.

1467 Supplemental File 19. U87 cell line species input file.

1468 Supplemental File 20. Gene Regulation input file for SPARCED-I model.

1469 Supplemental File 21. Gene Regulation input file for SPARCED-I Mechanism 1 model.

1470 Supplemental File 22. MCF10A RNA-seq and proteomics data.

1471 Supplemental File 23. SPARCED-I model SBML file.

1472 Supplemental File 24. SPARCED-I Mechanism 1 model SBML file. 
bioRxiv preprint doi: https://doi.org/10.1101/2020.11.09.373407; this version posted July 15,2021 . The copyright holder for this preprint (which was not certified by peer review) is the author/funder. All rights reserved. No reuse allowed without permission.

1473 Supplemental File 25. SPARCED-I Mechanism 2 model SBML file. 
A The mechanistic pan-cancer model

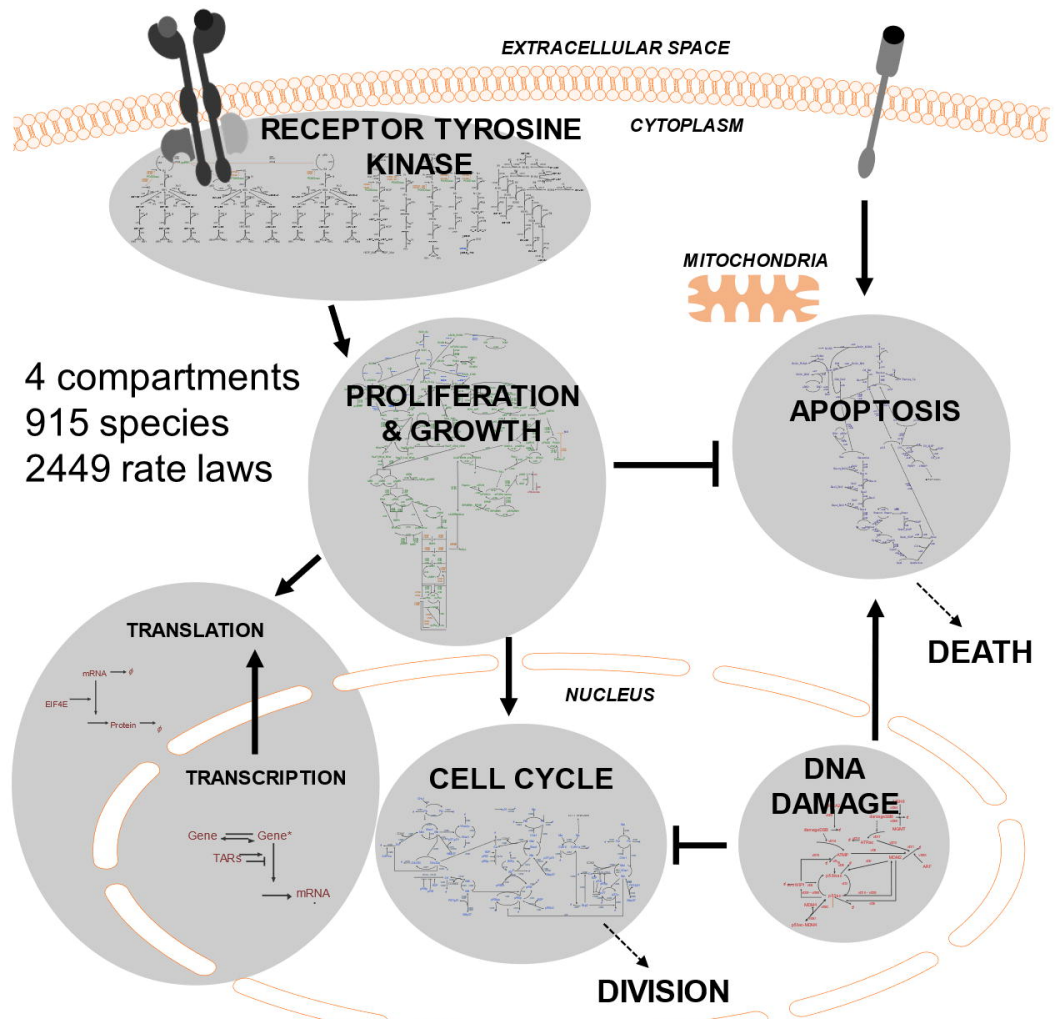

C

\begin{tabular}{|c|c|c|}
\hline & $\begin{array}{c}\text { Bouhaddou2018 } \\
\text { model }\end{array}$ & SPARCED model \\
\hline Input files & Model files & \\
\hline Simulation & MATLAB & \\
\hline MATLAB
\end{tabular}

B Model creation and simulation pipeline

- Omics data

- Species list

- Rate laws list

- Compartments list

- Stoichiometric matrix

- Transcriptional regulation matrix

- Observables matrix

\section{Input files}

- Structured

- Text \& numbers

- Easy to alter

- Annotated (GO terms, HGNC \& ENSEMBL identifiers)

\section{Model construction}

- createModel.ipynb

- Antimony (.txt) file

- SBML (.xml) file
- Minimally hardcoded scripts

- Open-source tools

- Human readable \& interpretable files

\section{Initialization}

- Initializer.txt

- initializeModel.ipynb

Context-specific model calibration

\section{Simulation}

\begin{tabular}{|c|c|}
\hline $\begin{array}{l}\text { - runModel.ipynb } \\
\text { - SGEmodule.py } \\
\text { - RunPrep.py } \\
\text { - AMICI package }\end{array}$ & $\begin{array}{l}\text { - runModel.py } \\
\text { - SGEmodule.py } \\
\text { - RunPrep.py } \\
\text { - AMICI package }\end{array}$ \\
\hline $\begin{array}{l}\text { Jupyter notebooks } \\
\text { implementation: } \\
\text { SPARCED-jupyter (Fig. }\end{array}$ & $\begin{array}{l}\text { Kubernetes+Nextflow } \\
\text { implementation: } \\
\text { SPARCED-nf (Fig. S1) }\end{array}$ \\
\hline
\end{tabular}




\section{SPARCED Model Creation}

These are the "default" input file names

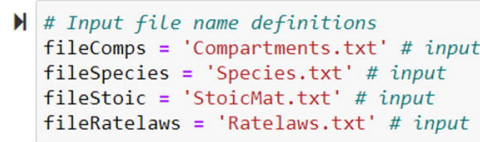

Input file processing and conversion to Antimony and SBML

The Model Compilation using AMICI package

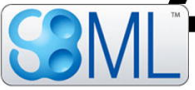

The model creation is now complete! Enjoy...

\section{Stochastic gene expression (SGEmodule.py)}

\section{Gene Switching - A Poisson process}

For each gene "gene ${ }_{i}$ :

- $q_{i} \sim$ Poisson.pmf $\left(k=0, m u=k G_{i} \cdot t s\right)$ \# kG are gene switching rate constants [40]

- $r_{i} \sim$ random. uniform $(0,1)$

- If gene $_{i}$ is "ON" and $r_{i}>q_{i} \rightarrow$ gene $_{i}$ is "OFF"

- If gene $_{i}$ is "OFF" and $r_{i}>q_{i} \rightarrow$ gene $_{i}$ is "ON"

\section{mRNA birth/death - Another Poisson process} For each $m R N A$ species " $m R_{j}$ ":

- $m R_{j_{\text {horn }}} \sim$ random.poisson $\left(v T C_{j} \cdot t s\right)$ \# VTC: transcription rate

- $\quad m R_{j_{\text {dead }}} \sim$ random.poisson $\left(v T C d_{j} \cdot t s\right)$ \# VTCd: transcript degradation rate

- $m R_{j_{(\text {nextTimePoint })}}=m R_{j}+m R_{j_{\text {born }}}-m R_{j_{\text {dead }}}$

$$
\text { F }
$$

\section{$\mathbf{F}$}

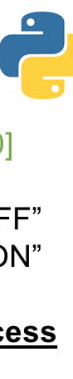

s

\section{Simulating} SPARCED model

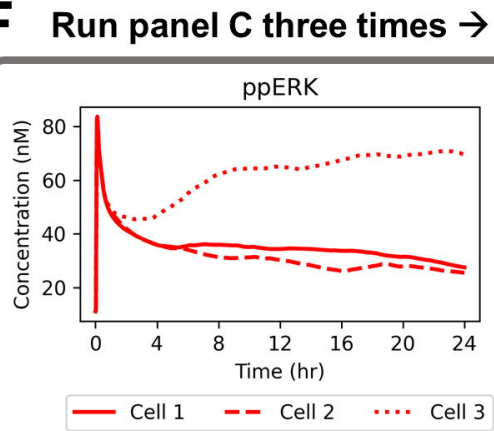

\section{SPARCED Model Initialization}

Load the model file, input files, and initialization specs

Repeat kTLadjustwhile( Species, observables, mRNAs, parameters, volumes)

\section{Simulating single cell responses using SPARCED}

\section{SPARCED Model Simulation}

The required user input -.> flagD, th, SBML file

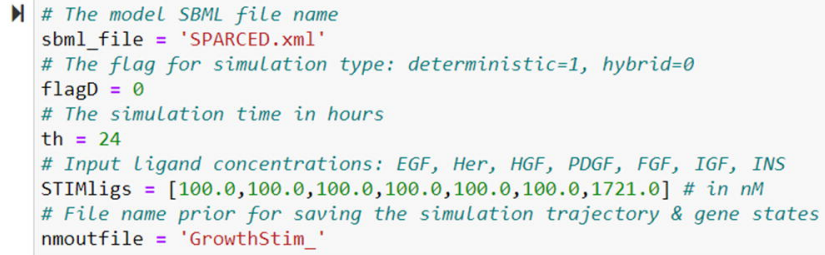

Species initial conditions --> ICs

Model import --> model object

Model simulation <-- flagD, th, SBML file, ICs, model

Y xoutS_all, xoutG_all, tout_all $=\operatorname{RunSPARCED}(f l a g D, t h, I C s,[]$, sbml_file, model $)$

D Hybrid simulation of SPARCED (RunSPARCED . PY)

SpeciesTraj $=[]$ \# Matrix to store species concentrations

GeneStatesTraj = [] \# Matrix to store gene states

\# Run 30sec (ts) simulations

- for each $\mathbf{3 0}$ sec (until final "th" is reached):

\# Update model mRNA values

- genestates,mRNAvals = SGEmodule $(\ldots)$

\# Run the next 30sec simulation:

- model.setInitialStates(mRNAIndDs,np.dot(mRNAvals))

- $\quad$ simdata = amici.runAmiciSimulation(model, solver)

\# Store the data:

- $\quad$ SpeciesTraj = np.vstack([SpeciesTraj, simdata(end,:)])

- GeneStatesTraj = np.vstack ([GeneStatesTraj, genestates])

\# check for cell death

- If [CPARP] > [PARP] :

- $\operatorname{print}($ 'Apoptosis happened')

- break 


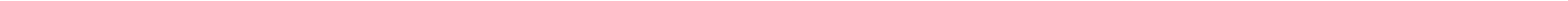


A

$+\mathrm{HS}+\mathrm{HS}$ Perturb cells

+ GFs - GFs (+EGF or +EGF+IFNG)
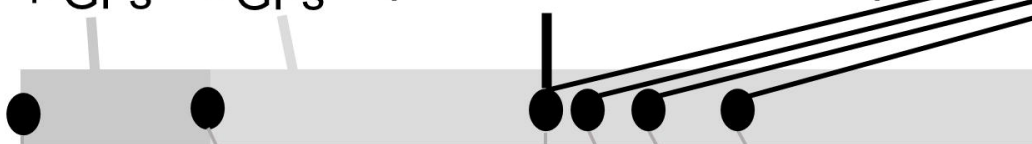

RPPA measurements

Experiments

$-24 \mathrm{hr} \quad-18 \mathrm{hr}$

$0148 \mathrm{hr}$

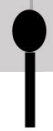

+ IGF

Perturb cells

(+EGF or +EGF+IFNG)

B

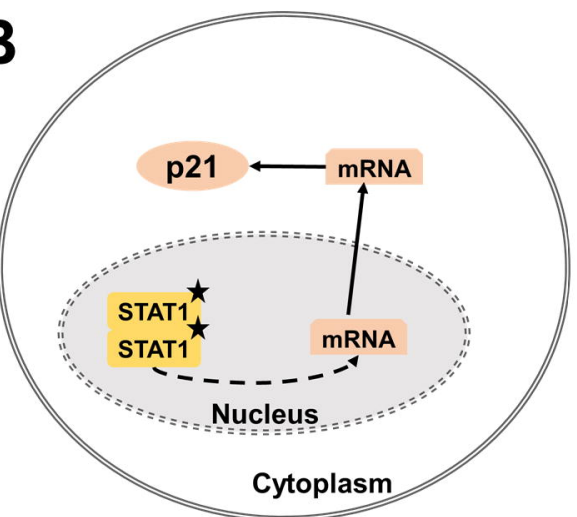

Mechanism 1: Activated STAT1 induces p21 transcription.

C

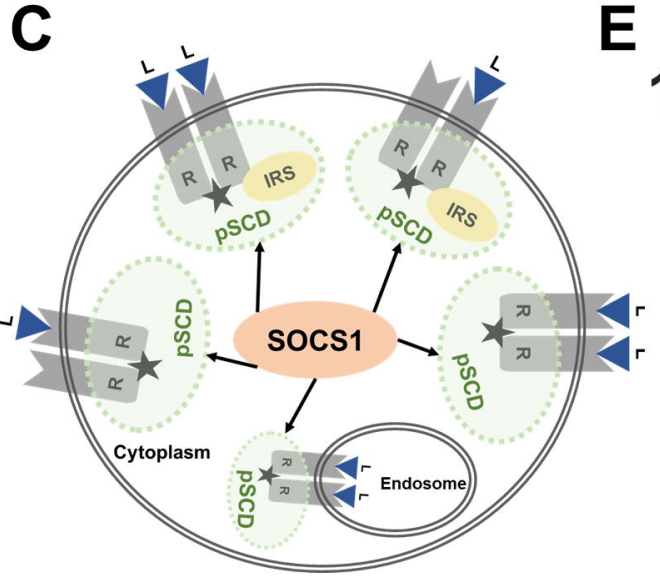

Mechanism 2: SOCS1 binds to "signaling competent receptor dimer" complexes (pSCD) containing species and sequesters them.
D

mech 1

100

50

0
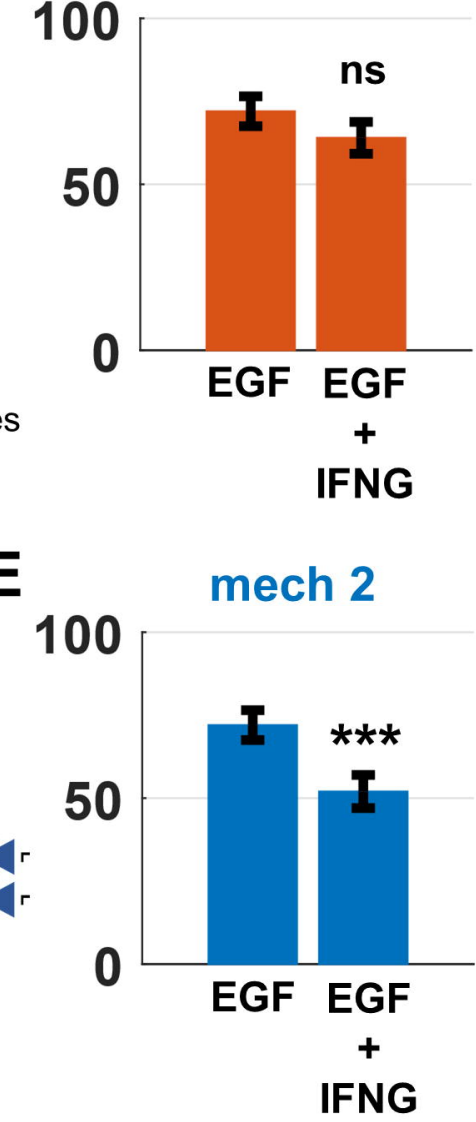

Number of total cell cycles @48 hrs
$24 \mathrm{hr}$

0.5
*HS: Horse serum

*GFs: Growth factors

(EGF+insulin)

$48 \mathrm{hr}$

Simulations

Count cyclin A

peaks

$\mathbf{F}$

mech 1, EGF+IFNG vs EGF

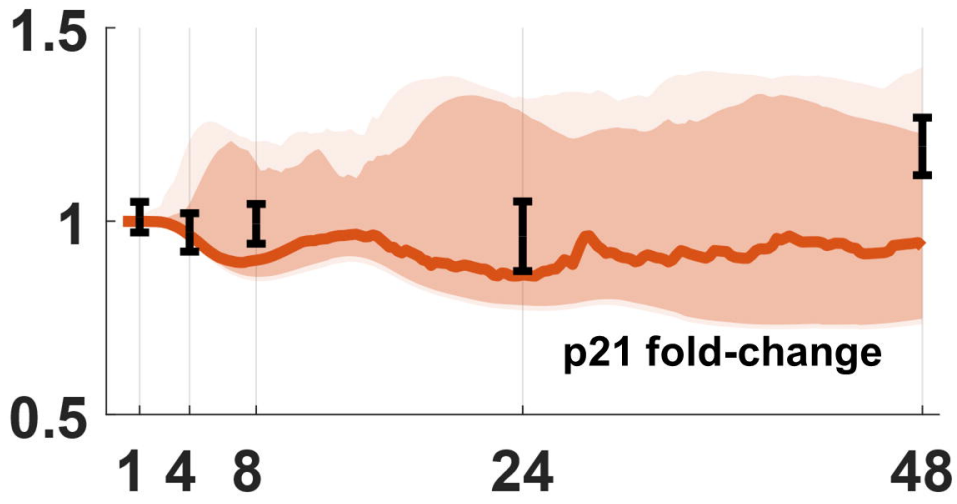

G mech 2, EGF+IFNG vs EGF

1.5

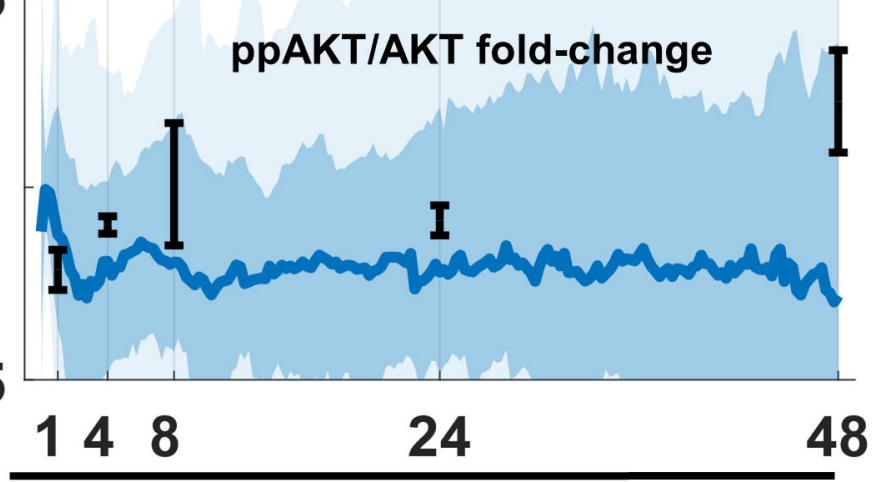

Time Post-Treatment (hours) Median cell trajectory $\mathbf{I}$ Exp. data Area plots: dark- $70^{\text {th }}$ quantile / light- $95^{\text {th }}$ quantile 